LAWRENCE LIVERMORE NAT IO N A L LABORATORY
Group Representations and Multinomial Combinatorics of the Icosahedral Symmetry

K. Balasubramanian

February 4, 2004

Molecular Physics 
This document was prepared as an account of work sponsored by an agency of the United States Government. Neither the United States Government nor the University of California nor any of their employees, makes any warranty, express or implied, or assumes any legal liability or responsibility for the accuracy, completeness, or usefulness of any information, apparatus, product, or process disclosed, or represents that its use would not infringe privately owned rights. Reference herein to any specific commercial product, process, or service by trade name, trademark, manufacturer, or otherwise, does not necessarily constitute or imply its endorsement, recommendation, or favoring by the United States Government or the University of California. The views and opinions of authors expressed herein do not necessarily state or reflect those of the United States Government or the University of California, and shall not be used for advertising or product endorsement purposes. 


\title{
Group Representations and Multinomial Combinatorics of the Icosahedral Symmetry
}

\author{
K. Balasubramanian* \\ Center for Image Processing and Integrated computing, University of California \\ Davis, Livermore, CA 94550; University of California \\ Chemistry and Material Science Directorate \\ Lawrence Livermore National Laboratory \\ Livermore, California 94550; \\ and Glenn T Seaborg Center, Lawrence Berkeley Laboratory, University of \\ California, Berkeley, CA 94720
}

The icosahedral symmetry is one of the most intriguing symmetries, as it not only presents challenge but it appears in many fullerenes and high energetic materials such as the dodecahedral $\mathrm{N}_{20}$. We have considered the combinatorics of all irreducible representations of the icosahedral symmetry for a number of multinomial partitions for vertex, face and edge colorings in this work. We have constructed the combinatorial tables for all irreducible representations for various multinomial partitions of colorings for the vertices, edge and faces of the icosahedron. These techniques should have important applications to enumerations and spectroscopy of fullerenes and high-energy materials such as $\mathrm{N}_{20}$.

\footnotetext{
*Address correspondence at kbala@ ucdavis.edu or fax:925-422-6810
} 


\section{Introduction.}

The icosahedral symmetry [1-19] is quite interesting and intriguing as it appears in fullerenes including the celebrated Buckminsterfullrene, $\mathrm{C}_{60}$ [1-5], high-energy materials such as

the dodecahedral $\mathrm{N}_{20}$ [7-12] and $\mathrm{C}_{60}$ analog, $\mathrm{C}_{48} \mathrm{~N}_{12}$ [13] and boron hydride species such as $\mathrm{B}_{12} \mathrm{H}_{12}{ }^{-2}$ [14], dodecahedral molecule $\mathrm{C}_{20} \mathrm{H}_{20}$ [6], which has been experimentally isolated [6] etc. The carbon-based fullerenes and related high-energy materials containing nitrogen have changed the earlier belief that five-fold axes do not occur commonly in structures. The study of icosahedral symmetry and its properties have become thus quite important and interesting. There have been several studies that were focused on the applications of combinatorics and graph theory to structures and spectroscopy [14-40]. These studies have been concerned with enumeration of isomers, chirality, spectroscopy, nuclear spin statistics, etc. There have been generalizations of combinatorial techniques beyond the celebrated Pólya's theorem to all irreducible representations [20] and by the current author to generalization of De Bruijn's theorem to all irreducible representations [25].

The techniques that have been presented in the literature, especially for the icosahedral symmetry, have been restricted to two or at most 3 kinds of substituents or colors or ligands. The combinatorics of applying all irreducible representations for multiple types of colors or ligands becomes complex not only due to many different types of partitions for such colors but also because of the combinatorial explosion that occurs, as the number of different types of colors goes up. Thus computing these numbers become quite challenging. When these techniques are applied to cases with 2 colors they become binomial expansions, and are much simpler. But the techniques rapidly evolve into multinomial combinatorial methods with rapidly growing coefficients for multiple ligands and colors. Thus a full combinatorial classification of these irreducible patterns requires exhaustive types of all possible distinct colors. For example, the vertices of an icosahedron can be colored with 12 different kinds of colors, and the edges of an icosahedron up to 30 different colors, and so on. The combinatorial complexity grows exponentially with the color partition, and the associated Young's diagram becomes complex with multiple rows. It seems that it would be quite interesting to have exhaustive tables at least for the vertex colorings as a function of Young's diagram for all irreducible representations of the icosahedron. Such generating functions can be quite useful for a number of applications that involve the icosahedral symmetry such as enumeration, chirality, spin statistics, and so on. For example, the naturally occurring ${ }^{209} \mathrm{Bi}$ has a 
nuclear spin of 9/2, which yields 10 distinct orientations for the magnetic spin orientations, given by $m_{\mathrm{f}}=-9 / 2,-7 / 2,-5 / 2,-3 / 2,-1 / 2,1 / 2,3 / 2,5 / 2,7 / 2$, and $9 / 2$. If each of these orientations is mapped into a distinct type of color the nuclear spin statistics of bismuth clusters with icosahedral symmetry requires multinomial combinatorics with 10 terms.

The objective of this study is to consider multinomial combinatorics of the icosahedron. By the application of combinatorial methods with irreducible representations, we have constructed exhaustive combinatorial tables for all irreducible representations of the icosahedral vertex colorings represented by the Young's diagrams or partitions of 12 for all irreducible representations, selected edge colorings and face colorings of the icosahedron. As a particular case, we have also discussed the chirality of the associated colorings of vertices, faces and edges of the icosahedron.

\section{Multinomial Combinatorics}

The basic combinatorial techniques use character values of the irreducible representations of the $\mathrm{I}_{\mathrm{h}}$ point group together with multinomial expansions. Multinomial expansions are constructed in terms of ordered partitions of $n$, denoted by, [n] into p parts (composition of the integer $\mathrm{n}$ into $\mathrm{p}$ parts) such that $n_{1} \geq 0, n_{2} \geq 0, \ldots ., n_{p} \geq 0, \quad \sum_{i=1}^{p} n_{i}=n$

A multinomial expansion in $\lambda$ 's is defined as

$$
\left(\lambda_{1}+\lambda_{2}+\ldots . \lambda_{p}\right)^{n}=\sum_{[\lambda]}\left(\begin{array}{lllll} 
& n & \\
n_{1} & n_{2} & . & . & n_{p}
\end{array}\right) \lambda_{1}^{n_{1}} \lambda_{2}^{n_{2}} \ldots \lambda_{1}^{n p}
$$

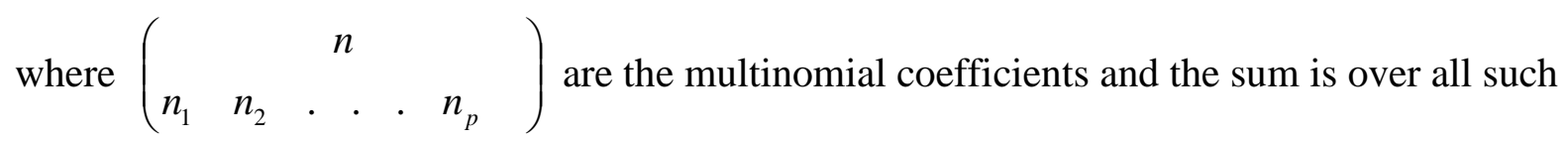
ordered partitions. The multinomial coefficients are computed as

$$
\left(\begin{array}{ccccc} 
& n & \\
n_{1} & n_{2} & \cdot & \cdot & \cdot \\
n_{p}
\end{array}\right)=\frac{n !}{n_{1} ! n_{2} ! \ldots \ldots n_{p} !}
$$

A useful result for computing the multinomial coefficients is 


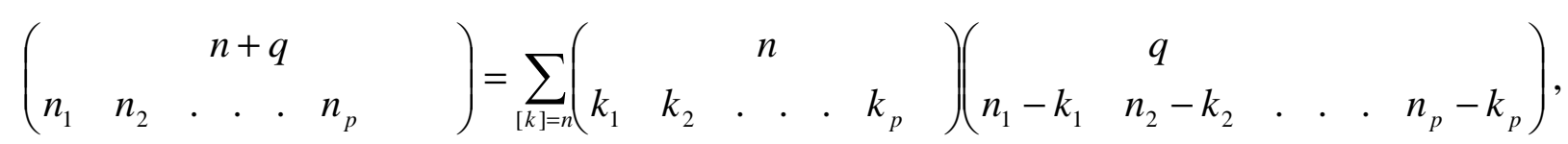

where $[\mathrm{k}]$ stands for all ordered partitions of $\mathrm{n}$ such that $\mathrm{k}_{1}+\mathrm{k}_{2}+\ldots .+\mathrm{k}_{\mathrm{p}}=\mathrm{n}$, with $\mathrm{k}_{\mathrm{i}}$ non-negative integers.

The present author [15,16,25] has generalized Pólya's theorem [23,24] to all irreducible representations of a group. This was proposed earlier by Williamson [31] and Merris [32] with a single group action but the present author provided a physical interpretation and generalized the technique to multiple group actions. In this technique a polynomial is constructed using the character values for each irreducible representation of the group. Suppose a symmetry operator $\mathrm{T}_{\mathrm{G}}{ }^{\chi}$ is constructed as

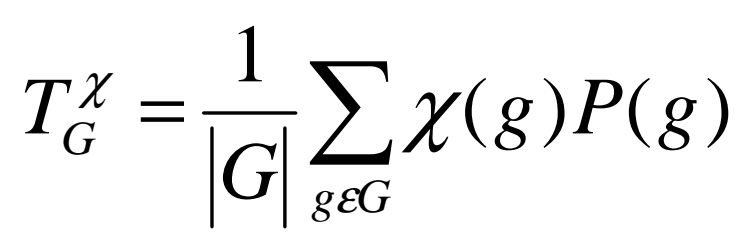

where $\chi(\mathrm{g})$ be the character value of $g \varepsilon \mathrm{G}$ for an irreducible representation $\Gamma$ in the group $\mathrm{G}$, and $\mathrm{P}(\mathrm{g})$ is a permutation operator for $\mathrm{g}$. A weighted permutation operator is obtained by introducing a weight $r$ for each kind of color in a set $\mathrm{R}$ with $\mathrm{D}$ being the set of vertices, edges or faces of the icosahedron. A coloring is simply a map $f$ from the set $\mathrm{D}$ to the set $\mathrm{R}$. The weight of a function $\mathrm{f}$ from $\mathrm{D}$ to $\mathrm{R}$ is given by

$$
W(f)=\prod_{i=1}^{n} w(f(i))
$$

We can define a permutational operator for each weight $\mathrm{W}$, and denote it by $\mathrm{P}_{\mathrm{W}}(\mathrm{g})$. In the most general form of matrix representations of $\mathrm{P}_{\mathrm{w}}(\mathrm{g})$, the tensor version of generalization of Pólya's theorem for all irreducible representations in the group would simply use the above matrix operators. However, a more convenient character version is obtained by considering the trace of $\mathrm{P}_{\mathrm{w}}(\mathrm{g})$ given by

$$
\operatorname{Tr}\left(P_{w}(g)\right)=\sum_{f}^{(g)} W(f),
$$

where the sum is over all $\mathrm{f}$ for which $\mathrm{gf}=\mathrm{f}$, and consequently, 


$$
\begin{aligned}
& T_{G}^{W, \chi}=\frac{1}{|G|} \sum_{g \varepsilon G} \chi(g) P_{W}(g) \\
& \operatorname{Tr}_{G}^{W, \chi}=\frac{1}{|G|} \sum_{g \varepsilon G} \chi(g) \operatorname{Tr}\left[P_{W}(g)\right]=\frac{1}{|G|} \sum_{g \varepsilon G} \chi(g) \sum_{f}^{(g)} W(f)
\end{aligned}
$$

The above expression is tantamount to replacement of every $\mathrm{s}_{\mathrm{k}}$ in the generalized character cycle index (GCCI) $\mathrm{P}_{\mathrm{G}}{ }^{\chi}$ for every irreducible representation as defined below:

$$
P_{G}^{\chi}=\frac{1}{|G|} \sum_{g \varepsilon G} \chi(g) s_{1}^{b_{1}} s_{2}^{b_{2}} \ldots . . s_{n}^{b_{n}}
$$

where the sum is over all elements of the group and $s_{1}^{b_{1}} s_{2}^{b_{2}} \ldots . . . s_{n}^{b_{n}}$ is the cyclic polynomial representation if $g$ in $G$ generates $b_{1}$ cycles of length $1, b_{2}$ cycles of length $2, \ldots . b_{n}$ cycles of length $\mathrm{n}$ when $\mathrm{g}$ acts on the set of elements $\mathrm{D}$. The difference between Pólya's cycle index and the one above is that for each irreducible representation, we have a cycle index (although may not be unique) due to the character values multiplying the polynomial. We carry out a multinomial replacement of every $s_{k}$ in the above expression by the following recipe. Suppose $\mathrm{n}$ is the number of elements in the set $\mathrm{D}$, and $\mathrm{R}$ consists of $\mathrm{n}$ distinct types of colors, for example, white, green, yellow, purple, magenta, green, red, and so on. Let $\lambda_{1}, \lambda_{2}, \ldots \lambda_{\mathrm{n}}$ be $\mathrm{n}$ distinct weights for these colors. The multinomial function is computed by replacing every $s_{k}$ in the above GCCI by $\lambda_{1}^{k}+\lambda_{2}^{k}+\ldots .+\lambda_{n}^{k}$. That is,

$$
G F^{\chi}=P_{G}^{\chi}\left(s_{k} \rightarrow \sum_{i} \lambda_{i}^{k}\right)
$$

The GF thus obtained above provides for generating functions for the various kinds of maps with different colorings such that each coloring transforms according to the irreducible representation $\Gamma$ whose character is $\chi$. For example, for the identity representation the GF enumerates isomers or equivalence classes. If $\Gamma$ is the anti-symmetric irreducible representation with -1 character values for all improper rotations, the GF enumerates all chiral isomers. In general $\mathrm{GF}^{\chi}$ enumerates 
equivalence classes of functions from $\mathrm{D}$ to $\mathrm{R}$ that transform according to the irreducible representation $\Gamma$.

As we showed above, the various terms in the GF have all ordered partitions of $\mathrm{n}$ into various parts given by Young's diagrams. Some of the terms in the complete generating function are combinatorially equivalent. To illustrate the terms $\lambda_{1}^{8} \lambda_{2}^{2} \lambda_{3} \lambda_{4}^{1}, \lambda_{1}^{8} \lambda_{3}^{2} \lambda_{4} \lambda_{6}^{1}, \lambda_{2}^{8} \lambda_{4}^{2} \lambda_{6} \lambda_{7}^{1}$, etc., are all equivalent as their coefficients in the multinomial expansion would be the same. The unique terms of the multinomial $\mathrm{GF}^{\chi}$ 's are the Young diagrams of partition $\mathrm{n}$ into various parts.

\section{Application to the Icosahedron.}

The icosahedral point group $\mathrm{I}_{\mathrm{h}}$ is probably the most complex among point groups exhibiting multiple axes of rotations, planes and improper axes of rotations. We start with the vertex colorings of the icosahedron with 12 different colors. In this case D is the set of 12 vertices of the icosahedron and $\mathrm{R}$ is a set of 12 distinct colors such as white, yellow, blue, green, red, purple, magenta, cyan, and so on. It is easy to show that there are $12^{12}$ possible vertex colorings of the icosahedron, which when symmetry-adapted would transform as various irreducible representations of the $I_{h}$ group. The number of totally symmetric representations is also the number of equivalence classes or positional isomers for the various vertex colorings.

Table 1 shows the GCCIs for vertex, face and edge colorings of the icosahedron. The character values are the same for all three cases; the main difference is the permutation representations for each conjugacy class depending on whether the set D is the set of vertices, edges or faces. For example, the cycle index polynomial for the $\mathrm{A}_{\mathrm{u}}$ irreducible representation of the $\mathrm{I}_{\mathrm{h}}$ group for the vertex colorings is given by

$$
P_{I_{h}}^{A_{u}}=\frac{1}{120}\left[s_{1}^{12}+24 s_{1}^{2} s_{5}^{2}+20 s_{3}^{4}+(15-1) s_{2}^{6}-24 s_{2}^{1} s_{10}-20 s_{6}^{2}-15 s_{1}^{4} s_{2}^{4}\right]
$$

If we replace every $\mathrm{s}_{\mathrm{k}}$ by $\sum_{i=1}^{12} \lambda_{i}^{k}$ in the above expression, we obtain, 


$$
\begin{aligned}
& P_{I_{h}}^{A_{u}}=\frac{1}{120}\left[\left(\lambda_{1}+\lambda_{2}+\ldots+\lambda_{12}\right)^{12}+24\left(\lambda_{1}+\lambda_{2}+\ldots+\lambda_{12}\right)^{2}\left(\lambda_{1}^{5}+\lambda_{2}^{5}+\ldots \lambda_{12}^{5}\right)^{2}+20\left(\lambda_{1}^{3}+\lambda_{2}^{3}+\ldots \lambda_{12}^{3}\right)^{4}\right. \\
& \left.+14\left(\lambda_{1}^{2}+\lambda_{2}^{2}+\ldots \lambda_{12}^{2}\right)^{6}-24\left(\lambda_{1}^{2}+\lambda_{2}^{2}+\ldots \lambda_{12}^{2}\right)\left(\lambda_{1}^{10}+\lambda_{2}^{10}+\ldots \lambda_{12}^{10}\right)-20\left(\lambda_{1}^{6}+\lambda_{2}^{6}+\ldots \lambda_{12}^{6}\right)^{2}\right] \\
& -15\left(\lambda_{1}+\lambda_{2}+\ldots+\lambda_{12}\right)^{4}\left(\lambda_{1}^{2}+\lambda_{2}^{2}+\ldots \lambda_{12}^{2}\right)^{4}
\end{aligned}
$$

The above expression can be simplified by collecting the coefficient of a typical term

$$
\lambda_{1}^{b_{1}} \lambda_{2}^{b_{2}} \ldots . \lambda_{12}^{b_{12}} \text { which enumerates number of times the irreducible representation } \mathrm{A}_{\mathrm{u}} \text { occurs }
$$

in the set of functions that contain $b_{1}$ colors of type $1, b_{2}$ colors of type $2, \ldots \ldots b_{12}$ colors of the type 12. Since the $A_{u}$ representation has all -1 character values for the improper rotations, it corresponds to the number of chiral vertex colorings of the icosahedron with the above distribution of colors.

Table 2 shows the full combinatorial results for the vertex colorings of the icosahedron for all irreducible representations and all partitions of 12 . In table 2, we show only the unique terms as determined by the Young diagrams for 12 . There is more than one term for each young diagram, for example, the term $1200 \ldots . .0$ could represent $\lambda_{1}^{12}$ or $\lambda_{2}^{12} \ldots . \lambda_{12}^{12}$. Thus each Young diagram represents multiple ordered partitions, and only a unique coefficient is shown in Table 2. As can be seen from table 2, the first non-zero binomial coefficient occurs for the $A_{u}$ representation for the partition [8+4], which has two chiral colorings. This would correspond to the term $\lambda_{1}^{8} \lambda_{2}^{4}$. The first nonzero trinomial coefficient occurs for the partition $[9+2+1]$ with 2 chiral colorings. The $\mathrm{T}_{1 \mathrm{~g}}$ representation has the first non-zero binomial term as $[9+3]$ and the first non-zero trinomial term as $[10+1+1]$. We can verify the correctness of all numbers using the fact that the sum of all numbers for each irreducible representation multiplied by the number of times that partition occurs for various irreducible representations in Table 2 should be equal to

$$
G F^{\chi}=P_{G}^{\chi}\left(s_{k} \rightarrow 12\right)
$$

For example, the above result for the $\mathrm{A}_{\mathrm{u}}$ representation gives

$$
\begin{aligned}
& P_{I_{h}}^{A_{\text {lu }}}=\frac{1}{120}\left[\left(12^{12}+24 \times 12^{2} \times 12^{2}+20 \times 12^{4}+14 \times 12^{6}-24 \times 12^{2}-20 \times 12^{2}-15 \times 12^{8}\right]\right. \\
& =8320953630
\end{aligned}
$$


Table 3 shows all such frequencies obtained directly by the above technique for all of the irreducible representations of the $\mathrm{I}_{\mathrm{h}}$ point group. It can also been that due to the fact that only the first term in the GF contributes to certain terms of the multinomial, and thus they can be readily obtained. For example, this appears to be the case for the last few rows for the vertex colorings according to the irreducible representation $\Gamma$. The last row is always given by

$$
\frac{1}{120}[12 \backslash \operatorname{dim}(\Gamma)]
$$

The one previous to the last row is given by

$$
\frac{1}{720}[12 \times \operatorname{dim}(\Gamma)]
$$

The last but one row is given by

$$
\frac{1}{480}[12 \times \operatorname{dim}(\Gamma)]
$$

Similar results can be obtained for a few other rows in Table 2, which have contributions only from the leading term.

Table 4 shows the face colorings of the icosahedron with up to 4 kinds of colors. We have restricted our table to 4 colors, as too many terms are generated for cases beyond 4 types of colors. As seen from Table 4, the first $\mathrm{A}_{\mathrm{u}}$ non-zero representation appears for the partition $18+2$ then $18+1+1$ both of which suggest that to produce a chiral structure out of an icosahedron by coloring the faces, one needs at least 18 colors of one kind and 2 colors of another kind for the binomial and 18,1,1 colorings for the trinomial. Analogous to the vertex colorings, the face colorings for the partition $1^{20}$ (not shown in table 4 ) is given by

$$
\frac{1}{120}[20 \ltimes \operatorname{dim}(\Gamma)] \text {. }
$$

Tables 5 and 6 show our edge colorings of the icosahedron for all the $\mathrm{g}$ and $\mathrm{u}$ irreducible representations, respectively for up to 4 types of colors as a function of partition of 30 as there are 30 edges for the icosahedron. The first non-zero binomial coloring occurs for the $28+2$ partition for the chiral representation $\mathrm{A}_{\mathrm{u}}$ with 3 colorings. The corresponding trinomial term occurs for $28+1+1$ with 6 chiral colorings. The corresponding tetranomial term with non-zero chiral coloring is 
attributed to the term $27+1+1+1$ with 200 chiral colorings. As seen from Tables 5 and 6 , the coefficients grow rapidly as a function of the complexity of the Young diagram, that is, as the number of rows of the diagram grows. We have used the quadruple precision arithmetic to compute these coefficients. Even though we do not show all of the results in tables 5 and 6 , the coefficient of the term with the $1^{30}$ partition with 30 rows for the Young diagram is given by

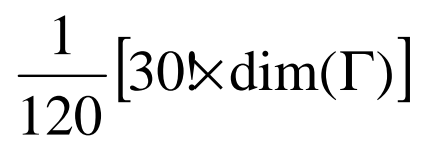

The generalized character cycle indices that we used to enumerate various colorings can also be used in other applications. For example, instead of the usual Pólya's substitution we can substitute in the icosahedral cycle indices other symmetric functions known as the S-functions [41]. For example, one may replace every $\mathrm{s}_{\mathrm{k}}$ by

$$
\lambda_{1}^{k} \lambda_{2}^{k}+\lambda_{1}^{k} \lambda_{3}^{k}+\ldots+\lambda_{1}^{k} \lambda_{n}^{k}+\lambda_{2}^{k} \lambda_{3}^{k}+\ldots .+\lambda_{2}^{k} \lambda_{3}^{k}+\lambda_{n-1}^{k} \lambda_{n}^{k}=\sum \lambda_{1}^{k} \lambda_{2}^{k}
$$

in the generalized cycle indices to produce interesting new combinatorial identities for each irreducible representation. We may also consider the replacement by tri-symmetric functions of the kind $\sum \lambda_{1}^{k} \lambda_{2}^{k} \lambda_{3}^{k}$ where the sum involves all possible symmetric combinations with three terms at a time. It can be seen that the method could be generalized to a multinomial S-function.

The enumeration of electronic configurations can be achieved [30] by choosing weights 1 , $\mathrm{w}, \mathrm{w}^{2} \ldots \mathrm{w}^{\mathrm{n}}$. If we stop with weights $1, \mathrm{w}$, and $\mathrm{w}^{2}$ this corresponds to filling the orbitals with zero, 1 or 2 electrons as by Pauli exclusion principle only as at most 2 electrons are allowed in any orbital. For example, the GF for the $\mathrm{A}_{\mathrm{g}}$ representation of the vertex group of icosahedron for the general weights is given by

$$
\begin{aligned}
& P_{I_{h}}^{A_{g}}=\frac{1}{120}\left[\left(1+w+w^{2}+w^{3}+\ldots w^{12}\right)^{12}+24\left(1+w+w^{2}+w^{3}+\ldots w^{12}\right)^{2}\left(1+w^{5}+w^{10}+w^{15}+\ldots w^{60}\right)^{2}+\right. \\
& +20\left(1+w^{3}+w^{6}+w^{9}+\ldots w^{36}\right)^{6}+16\left(1+w^{2}+w^{4}+w^{8}+\ldots w^{24}\right)^{6}-24\left(1+w^{2}+w^{4}+w^{8}+\ldots w^{24}\right) \\
& \left(1+w^{10}+w^{20}+w^{30}+\ldots w^{120}\right)-20\left(1+w^{6}+w^{12}+w^{18}+\ldots w^{72}\right)^{2}-15\left(1+w+w^{2}+w^{3}+\ldots w^{12}\right)^{12} \\
& \times\left(1+w^{2}+w^{4}+w^{8}+\ldots w^{24}\right)^{4}
\end{aligned}
$$


Other variations that lead to infinite power series in multinomial weights are also feasible. There is much to be desired in such powerful multinomial combinatorial theory.

A large number of applications of multinomial combinatorics of the kind developed here is possible. One of the important applications would be to nuclear spin statistics of molecular rovibronic levels [14-16,20]. Clusters of many species such as bismuth clusters exhibit high spin states thus spanning a multiple set of nuclear spin projections. Each projection becomes a variable in the multinomial combinatorial expansion. For example, nuclear spin properties of bismuth clusters would require such elaborate multivariable combinatorial expansions. Many inorganic species exhibit multiple ligands. If each type of ligand were to be associated with a type of color then enumeration of possible ligand substitutions for the icosahedron would become a multinomial problem with ligand partitions represented by the various Young diagrams.

Longuet-Higgins [42] has formulated the symmetry groups of non-rigid molecules as permutation-inversion groups. The rovibronic levels and nuclear spin statistical weights of nonrigid molecules and clusters would require multinomial combinatorial representations if the nuclei in the cluster have high-spin characteristics. The rovibronic levels of such species are split into tunneling levels as the non-rigid cluster tunnels through surmountable potential minima. The nuclear spin statistical weights of the tunneling levels would require multinomial polynomial expansions in terms of the character representations of the permutation-inversion groups of nonrigid molecules. Thus many applications seem to be feasible to molecular physics.

\section{Conclusion}

In this work we have obtained the full combinatorial tables for all irreducible representations of the icosahedral group for the vertex, edge and face colorings of the icosahedron using multinomial combinatorics. The tables provided full enumerations for all possible vertex colorings that transform according to the various irreducible representations of the $\mathrm{I}_{\mathrm{h}}$ group in terms of partitions or the young diagram. The results also included the chiral edge, vertex, and face colorings for all young diagrams. The results for other irreducible representations were interpreted. We have shown that there is rich combinatorics in the GCCIs when replacements are made for each term by a multinomial of S-functions. 


\section{Acknowledgement}

This research was performed under the auspices of the US department of Energy by the University of California, LLNL under contract number W-7405-Eng-48 while the work at UC Davis was supported by National Science Foundation.

\section{References}

[1] Kroto, H. W., Heath, J. R., O’Brien, S.C., Curl, R. F., and Smalley, R. E., 1985, Nature, 318, 162.

[2] Curl, R. F. and Smalley, R. E., 1988, Science, 242, 1017.

[3] Kroto, H. W., 1989, Comput Math. Appl. 17, 417.

[4] Kroto, H.W., Allaf, A. W., and Blam, S. P., 1989, Chem. Rev. 91, 1213.

[5] Chibante, L. P. F., and Smalley, R. E., 1992, Complete Buckminterfullerence Bibliography.

[6] Paquette, L. A., 1989, Chem. Rev., 89, 1051.

[7] Bliznyuk, A. A., Shen, M., and Schaefer III, H. F., 1992, Chem. Phys. Letters 198, 249.

[8] Seidl, E. T., and Schaefer III, H. F., 1988, J. Chem. Phys. 88, 7043.

[9] Balasubramanian, K., 1993, Chem. Phys. Lett. 202, 272.

[10] Alkorta, I., Elguero, J., Rozas, I., and Balaban, A. T., 1991, J. Mol. Struct. 228, 47.

[11] Schulman, J. M. and Disch, R.J., 1978, J. Am. Chem. Soc., 100, 5677.

[12] Chen, C., Lu, L.-H., and Wang, Y.-W., 1992, J. Mol. Struc., 253, 1.

[13] Manaa, M. R.; Sprehn, D. W.; Ichord, H. A., 2002, J. Am. Chem. Soc.124, 13990.

[14] Balasubramanian, K., Strauss, H., and Pitzer, K. S., 1982, J. Mol. Spectrosc.

[15] Balasubramanian, K., 1991, Chem. Phys. Lett. 183, 292.

[16] Balasubramanian, K., 1992, Chem. Phys. Lett. 200, 649.

[17] Colpa, J. P., and Temme, F., 1991, Z. Phys. D. 23, 187.

[18] Harter, W. G., and Weeks, D. E., 1989, J. Chem. Phys. 90, 4727

[19] Harter, W. G., and Reimer, T. C., 1992, J. Chem. Phys. 194, 230; 198, 429E.

[20] Balasubramanian, K., 1985, Chemical Rev. 85, 599.

[21] Balasubramanian, K., 1979, Theor. Chim. Acta, 51, 37

[22] Balaban, A. T., 1976, “Chemical Applications of Graph Theory”, Academic, New York, NY

[23] Pólya, G., and Read, R.C., 1987, “Combinatorial Enumeration of Groups, Graphs, and Chemical Compounds”, Springer, New York and Berlin. 
[24] Pólya, G., 1937, Acta. Math. 68, 145.

[25] Balasubramanian, K., 1993, J. Math Chem. 14, 113

[26] Robinson, R. W., Harary, F. and Balaban, A. T., 1976, Tetrahedron 32,355.

[27] Rouvray, D. H., 1974, Chem. Soc. Rev. 3, 355.

[28] Harary, F. and Palmer, E. M., 1973, “Graphical Enumeration”, Academic press, New York, NY

[29] Balasubramanian, K., 1981, J. Chem. Phys., 74, 6824

[30] Balasubramanian, K., 1981, Int. J. Quant. Chem. 20, 1255

[31] Williamson, S. G., 1971, J. Comb. Theory, 11, 122

[32] Merris, R., 1980, Linear Algebra and Applications, 29, 255.

[33] King, R. B. 1987 J. Math. Chem. 1, 15.

[34] King R. B., 1987, J. Math. Chem. 1, 55.

[35] King, R. B., 2003, in Chemical Explanation: Characteristics, Development, Autonomy, ed. Earley, J. E., Ann. N. Y. Acad. Sci., 988, 158

[36] Balasubramanian, K., 1991, Chemical Physics Letters, 183,292

[37] Balasubramanian, K., 1991, J. Chem. Phys, 95, 8273.

[38] Balasubramanian, K., 1993, Chemical Physics Lett. 210, 153.

[39] Balasubramanian, K., 1992, Chemical Physics Lett. 200, 649.

[40] Balasubramanian, K., 1996, Chem. Phys. Lett. 260, 476

[41] Littlewood, D. E., 1940, "Theory of Group Representation and Matrix Product of Groups", Clarendon, Oxford.

[42] Longuet-Higgins, H.C., 1963, Molecular Physics 6, 445. 
Table 1 GCCI table for the vertex/face/edge colorings of the icosahedron

\begin{tabular}{|l|l|l|l|l|l|l|l|l|}
\hline Vertex & $1^{12}$ & $1^{2} 5^{2}$ & $3^{4}$ & $2^{6}$ & $2^{6}$ & 210 & $6^{2}$ & $1^{4} 2^{4}$ \\
\hline Face & $1^{20}$ & $5^{4}$ & $1^{2} 3^{6}$ & $2^{10}$ & $2^{10}$ & $10^{2}$ & $26^{3}$ & $1^{4} 2^{8}$ \\
\hline Edge & $1^{30}$ & $5^{6}$ & $3^{10}$ & $1^{2} 2^{14}$ & $2^{15}$ & $10^{3}$ & $6^{5}$ & $1^{4} 2^{13}$ \\
\hline Order & 1 & 24 & 20 & 15 & 1 & 24 & 20 & 15 \\
\hline $\mathrm{A}_{\mathrm{g}}$ & 1 & 1 & 1 & 1 & 1 & 1 & 1 & 1 \\
\hline $\mathrm{A}_{\mathrm{u}}$ & 1 & 1 & 1 & 1 & -1 & -1 & -1 & -1 \\
\hline $\mathrm{T}_{1 \mathrm{~g}}=\mathrm{T}_{2 \mathrm{~g}}$ & 3 & $1 / 2$ & 0 & -1 & 3 & $1 / 2$ & 0 & -1 \\
\hline $\mathrm{T}_{1 \mathrm{u}}=\mathrm{T}_{2 \mathrm{u}}$ & 3 & $1 / 2$ & 0 & -1 & -3 & $-1 / 2$ & 0 & 1 \\
\hline $\mathrm{G}_{\mathrm{g}}$ & 4 & -1 & 1 & 0 & 4 & -1 & 1 & 0 \\
\hline $\mathrm{G}_{\mathrm{u}}$ & 4 & -1 & 1 & 0 & -4 & 1 & -1 & 0 \\
\hline $\mathrm{H}_{\mathrm{g}}$ & 5 & 0 & -1 & 1 & 5 & 0 & -1 & 1 \\
\hline $\mathrm{H}_{\mathrm{u}}$ & 5 & 0 & -1 & 1 & -5 & 0 & 1 & -1 \\
\hline
\end{tabular}


Table 2 Icosahedral vertex combinatorics for all irreducible representations up to 12 colors

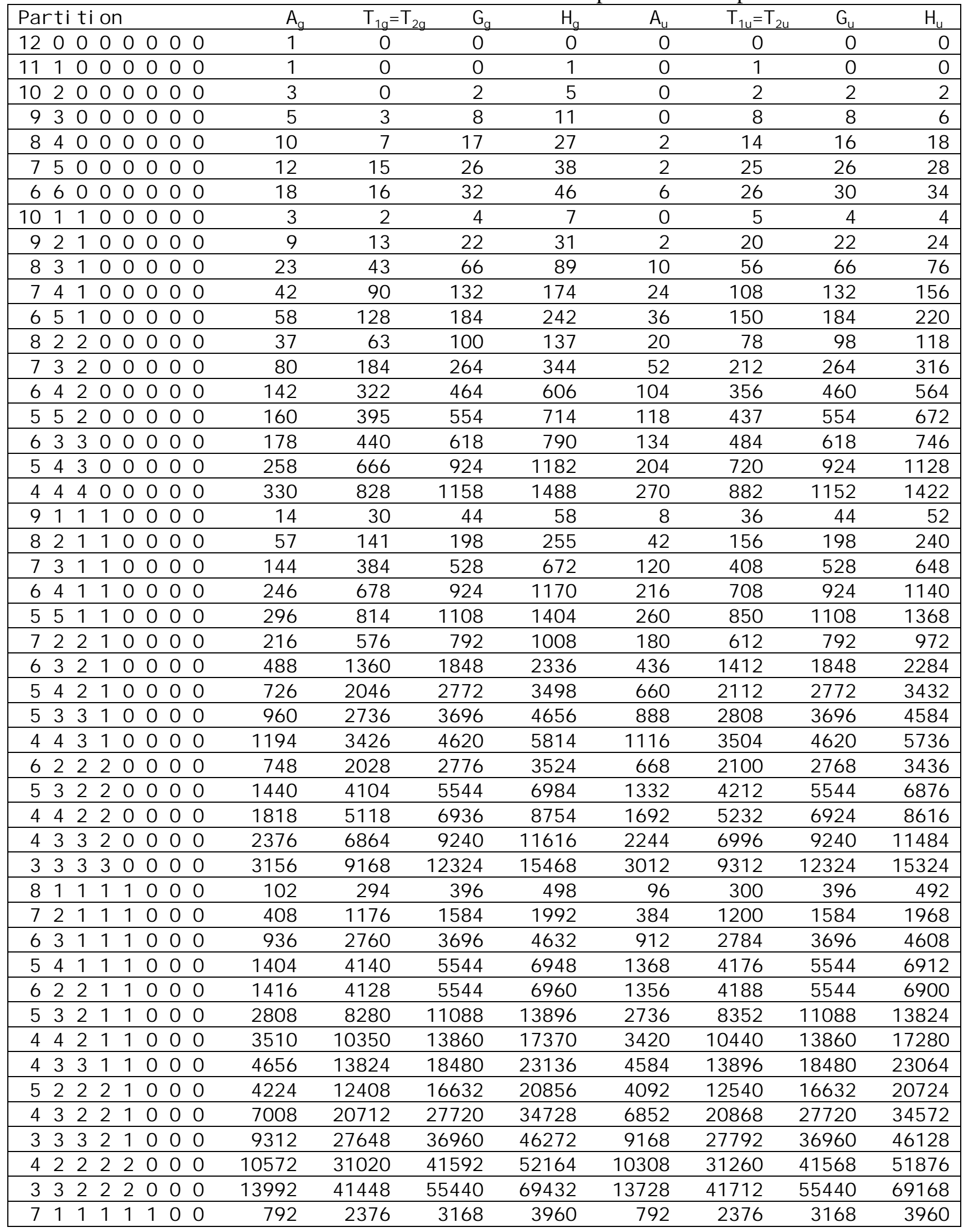




\begin{tabular}{|c|c|c|c|c|c|c|c|c|}
\hline $\begin{array}{llllllll}6 & 2 & 1 & 1 & 1 & 1 & 0 & 0\end{array}$ & 2784 & 8304 & 11088 & 13872 & 2760 & 8328 & 11088 & 13848 \\
\hline $\begin{array}{lllllllll}5 & 3 & 1 & 1 & 1 & 1 & 0 & 0\end{array}$ & 5544 & 16632 & 22176 & 27720 & 5544 & 16632 & 22176 & 27720 \\
\hline $\begin{array}{llllllll}4 & 4 & 1 & 1 & 1 & 1 & 0 & 0\end{array}$ & 6948 & 20772 & 27720 & 34668 & 6912 & 20808 & 27720 & 34632 \\
\hline $\begin{array}{lllllllll}5 & 2 & 2 & 1 & 1 & 1 & 0 & 0\end{array}$ & 8352 & 24912 & 33264 & 41616 & 8280 & 24984 & 33264 & 41544 \\
\hline $\begin{array}{llllllll}4 & 3 & 2 & 1 & 1 & 1 & 0 & 0\end{array}$ & 13896 & 41544 & 55440 & 69336 & 13824 & 41616 & 55440 & 69264 \\
\hline $\begin{array}{lllllllll}3 & 3 & 3 & 1 & 1 & 1 & 0 & 0\end{array}$ & 18480 & 55440 & 73920 & 92400 & 18480 & 55440 & 73920 & 92400 \\
\hline $\begin{array}{llllllll}4 & 2 & 2 & 2 & 1 & 1 & 0 & 0\end{array}$ & 20880 & 62280 & 83160 & 104040 & 20700 & 62460 & 83160 & 103860 \\
\hline $\begin{array}{llllllll}3 & 3 & 2 & 2 & 1 & 1 & 0 & 0\end{array}$ & 27792 & 83088 & 110880 & 138672 & 27648 & 83232 & 110880 & 138528 \\
\hline 322222100 & 41736 & 124584 & 166320 & 208056 & 41424 & 124896 & 166320 & 207744 \\
\hline 22222200 & 62736 & 186768 & 249504 & 312240 & 62184 & 187272 & 249456 & 311640 \\
\hline $\begin{array}{llllllll} & 1 & 1 & 1 & 1 & 1 & 1 & 0\end{array}$ & 5544 & 16632 & 22176 & 27720 & 5544 & 16632 & 22176 & 27720 \\
\hline $\begin{array}{llllll}1 & 1 & 1 & 1 & 1 & 0\end{array}$ & 16632 & 49896 & 66528 & 83160 & 16632 & 49896 & 66528 & 83160 \\
\hline $\begin{array}{llllllll}4 & 3 & 1 & 1 & 1 & 1 & 1 & 0\end{array}$ & 27720 & 83160 & 110880 & 138600 & 27720 & 83160 & 110880 & 138600 \\
\hline $\begin{array}{lllllllll}4 & 2 & 2 & 1 & 1 & 1 & 1 & 0\end{array}$ & 41616 & 124704 & 166320 & 207936 & 41544 & 124776 & 166320 & 207864 \\
\hline $\begin{array}{lllllllll}3 & 3 & 2 & 1 & 1 & 1 & 1 & 0\end{array}$ & 55440 & 166320 & 221760 & 277200 & 55440 & 166320 & 221760 & 277200 \\
\hline 222111 & 83232 & 249408 & 332640 & 415872 & 83088 & 249552 & 332640 & 415728 \\
\hline 222221 & 124920 & 374040 & 498960 & 623880 & 124560 & 374400 & 498960 & 623520 \\
\hline $\begin{array}{llllllll}5 & 1 & 1 & 1 & 1 & 1 & 1 & 1\end{array}$ & 33264 & 99792 & 133056 & 166320 & 33264 & 99792 & 133056 & 166320 \\
\hline $\begin{array}{llllllll}4 & 2 & 1 & 1 & 1 & 1 & 1 & 1\end{array}$ & 83160 & 249480 & 332640 & 415800 & 83160 & 249480 & 332640 & 415800 \\
\hline $\begin{array}{llllll}1 & 1 & 1 & 1 & 1 & 1\end{array}$ & 110880 & 332640 & 443520 & 554400 & 110880 & 332640 & 443520 & 554400 \\
\hline $\begin{array}{lllllll}3 & 2 & 2 & 1 & 1 & 1 & 1\end{array}$ & 166320 & 498960 & 665280 & 831600 & 166320 & 498960 & 665280 & 831600 \\
\hline $\begin{array}{llllllll}2 & 2 & 2 & 2 & 1 & 1 & 1 & 1\end{array}$ & 249552 & 748368 & 997920 & 1247472 & 249408 & 748512 & \multicolumn{2}{|c|}{9979201247328} \\
\hline $\begin{array}{llllllllll}4 & 1 & 1 & 1 & 1 & 1 & 1 & 1 & 1\end{array}$ & 166320 & 498960 & 665280 & 831600 & 166320 & 498960 & \multicolumn{2}{|c|}{665280831600} \\
\hline $\begin{array}{llllllllll} & 2 & 1 & 1 & 1 & 1 & 1 & 1 & 1\end{array}$ & 332640 & 997920 & 1330560 & 1663200 & 332640 & 997920 & \multicolumn{2}{|c|}{13305601663200} \\
\hline $\begin{array}{llllllllll}2 & 2 & 2 & 1 & 1 & 1 & 1 & 1 & 1 \\
\end{array}$ & 498960 & 1496880 & 1995840 & 2494800 & 498960 & 1496880 & \multicolumn{2}{|c|}{19958402494800} \\
\hline \begin{tabular}{|llllllllll}
2 & 2 & 1 & 1 & 1 & 1 & 1 & 1 & 1 & 1
\end{tabular} & 997920 & 2993760 & 3991680 & 4989600 & 997920 & 2993760 & \multicolumn{2}{|c|}{$\begin{array}{l}1993040<494800 \\
3991680 \quad 4989600\end{array}$} \\
\hline $\begin{array}{llllllll} & 1 & 1 & 1 & 1 & 1 & 1 & 1 \\
\end{array}$ & & & & & & & \multicolumn{2}{|c|}{26611203326400} \\
\hline 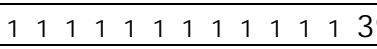 & & & & & & & & \\
\hline
\end{tabular}


Table 3 Frequencies of all irreducible representations in vertex edge combinatorics of icosahedron for all 12 colors.

\begin{tabular}{|l|l|}
\hline $\mathrm{A}_{\mathrm{g}}$ & 8345970370 \\
\hline $\mathrm{A}_{\mathrm{u}}$ & 8320953630 \\
\hline $\mathrm{T}_{1 \mathrm{~g}}=\mathrm{T}_{2 \mathrm{~g}}$ & 24987401010 \\
\hline $\mathrm{G}_{\mathrm{g}}$ & 33333366330 \\
\hline $\mathrm{H}_{\mathrm{g}}$ & 41679331650 \\
\hline $\mathrm{T}_{1 \mathrm{u}}=\mathrm{T}_{2 \mathrm{u}}$ & 25012350990 \\
\hline $\mathrm{G}_{\mathrm{u}}$ & 33333299670 \\
\hline $\mathrm{H}_{\mathrm{u}}$ & 41654248350 \\
\hline
\end{tabular}

${ }^{a}$ Sum of frequencies $x$ dimension of the reps for all irreducible reps is verified to be $12^{12}$. 
Table 4 Icosahedral Face Combinatorics for all irreducible representations up to 4 colors

\begin{tabular}{|c|c|c|c|c|c|c|c|c|c|c|}
\hline 20 & 00 & 0 & 1 & 0 & 0 & 0 & 0 & 0 & 0 & 0 \\
\hline 19 & 10 & 0 & 1 & 0 & 1 & 1 & 0 & 1 & 1 & 0 \\
\hline 18 & 20 & 0 & 5 & 2 & 7 & 11 & 1 & 5 & 6 & 7 \\
\hline 17 & 30 & 0 & 15 & 24 & 39 & 51 & 6 & 33 & 39 & 42 \\
\hline 16 & 40 & 0 & 58 & 107 & 165 & 217 & 38 & 124 & 162 & 194 \\
\hline 15 & 50 & 0 & 149 & 370 & 517 & 663 & 113 & 406 & 517 & 627 \\
\hline 14 & 60 & 0 & 371 & 928 & 1299 & 1661 & 310 & 980 & 1290 & 1594 \\
\hline 13 & 70 & 0 & 693 & 1896 & 2589 & 3267 & 609 & 1980 & 2589 & 3183 \\
\hline 12 & 80 & 0 & 1135 & 3074 & 4209 & 5335 & 1022 & 3172 & 4194 & 5210 \\
\hline 11 & 90 & 0 & 1466 & 4136 & 5602 & 7058 & 1340 & 4262 & 5602 & 6932 \\
\hline 10 & 10 & 0 & 1648 & 4528 & 6172 & 7800 & 1510 & 4648 & 6156 & 7646 \\
\hline 18 & $\begin{array}{ll}1 & 1\end{array}$ & 10 & 5 & 8 & 13 & 17 & 2 & 11 & 13 & 14 \\
\hline 17 & 21 & 10 & 34 & 80 & 114 & 148 & 23 & 91 & 114 & 137 \\
\hline 16 & 31 & 10 & 176 & 472 & 648 & 818 & 151 & 497 & 648 & 793 \\
\hline 15 & 41 & 10 & 674 & 1912 & 2586 & 3254 & 622 & 1964 & 2586 & 3202 \\
\hline 14 & 51 & 10 & 1984 & 5768 & 7752 & 9736 & 1892 & 5860 & 7752 & 9644 \\
\hline 13 & 61 & 10 & 4597 & 13496 & 18093 & 22675 & 4457 & 13636 & 18093 & 22535 \\
\hline 12 & 71 & 10 & 8501 & 25096 & 33597 & 42083 & 8305 & 25292 & 33597 & 41887 \\
\hline 11 & 81 & 10 & 12716 & 37672 & 50388 & 63104 & 12478 & 37910 & 50388 & 62866 \\
\hline 10 & $\begin{array}{ll}9 & 1 \\
\end{array}$ & 10 & 15536 & 46056 & 61592 & 77108 & 15270 & 46322 & 61592 & 76842 \\
\hline 16 & 22 & 20 & 274 & 698 & 972 & 1246 & 233 & 733 & 966 & 1199 \\
\hline 15 & 32 & 20 & 1337 & 3832 & 5169 & 6503 & 1249 & 3920 & 5169 & 6415 \\
\hline 14 & 42 & 20 & 4984 & 14408 & 19392 & 24376 & 4796 & 14572 & 19368 & 24164 \\
\hline 13 & 52 & 20 & 13720 & 40544 & 54264 & 67984 & 13412 & 40852 & 54264 & 67676 \\
\hline 12 & 62 & 20 & 29739 & 87864 & 117603 & 147333 & 29262 & 88284 & 117546 & 146802 \\
\hline 11 & 72 & 20 & 50696 & 150856 & 201552 & 252248 & 50080 & 151472 & 201552 & 251632 \\
\hline 10 & 82 & 20 & 69812 & 207364 & 277176 & 346988 & 69070 & 208022 & 277092 & 346162 \\
\hline 9 & 92 & 20 & 77370 & 230560 & 307930 & 385290 & 76600 & 231330 & 307930 & 384520 \\
\hline 14 & 33 & 30 & 6557 & 19288 & 25845 & 32387 & 6373 & 19472 & 25845 & 32203 \\
\hline 13 & 43 & 30 & 22802 & 67648 & 90450 & 113222 & 22438 & 68012 & 90450 & 112858 \\
\hline 12 & 53 & 30 & 59085 & 176064 & 235149 & 294219 & 58497 & 176652 & 235149 & 293631 \\
\hline 11 & 63 & 30 & 118002 & 352296 & 470298 & 588270 & 117162 & 353136 & 470298 & 587430 \\
\hline 10 & 73 & 30 & 185308 & 553736 & 739044 & 924292 & 184244 & 554800 & 739044 & 923228 \\
\hline 9 & 83 & 30 & 231550 & 692240 & 923790 & 1155310 & 230360 & 693430 & 923790 & 1154120 \\
\hline 12 & 44 & 40 & 74014 & 219968 & 293982 & 367966 & 73286 & 220612 & 293898 & 367154 \\
\hline 11 & 54 & 40 & 176904 & 528528 & 705432 & 882336 & 175812 & 529620 & 705432 & 881244 \\
\hline 10 & 64 & 40 & 324428 & 968968 & 1293396 & 1617764 & 322888 & 970340 & 1293228 & 1616056 \\
\hline 9 & 74 & 40 & 462820 & 1384760 & 1847580 & 2310340 & 461000 & 1386580 & 1847580 & 2308520 \\
\hline 8 & 84 & 40 & 521000 & 1557610 & 2078610 & 2599610 & 519040 & 1559360 & 2078400 & 2597440 \\
\hline 10 & 55 & 50 & 388788 & 1163166 & 1551948 & 1940736 & 387192 & 1164762 & 1551948 & 1939140 \\
\hline 9 & 65 & 50 & 647706 & 1938888 & 2586594 & 3234270 & 645606 & 1940988 & 2586594 & 3232170 \\
\hline 8 & 75 & 50 & 832592 & 2493016 & 3325608 & 4158200 & 830212 & 2495396 & 3325608 & 4155820 \\
\hline 8 & 66 & 50 & 971840 & 2908192 & 3880032 & 4851824 & 969178 & 2910572 & 3879750 & 4848886 \\
\hline 7 & 76 & 50 & 1109966 & 3324208 & 4434174 & 5544050 & 1107166 & 3327008 & 4434174 & 5541250 \\
\hline 17 & 11 & 1 & 60 & 168 & 228 & 288 & 54 & 174 & 228 & 282 \\
\hline 16 & 21 & 1 & 498 & 1440 & 1938 & 2436 & 471 & 1467 & 1938 & 2409 \\
\hline 15 & 31 & 1 & 2610 & 7728 & 10338 & 12942 & 2562 & 7776 & 10338 & 12894 \\
\hline 14 & 41 & 1 & 9744 & 29016 & 38760 & 48504 & 9636 & 29124 & 38760 & 48396 \\
\hline 13 & 51 & 1 & 27216 & 81312 & 108528 & 135744 & 27048 & 81480 & 108528 & 135576 \\
\hline 12 & 61 & 1 & 58917 & 176232 & 235149 & 294051 & 58665 & 176484 & 235149 & 293799 \\
\hline 11 & 77 & 1 & 100944 & 302160 & 403104 & 504048 & 100608 & 302496 & 403104 & 503712 \\
\hline 10 & 81 & 1 & 138756 & 415512 & 554268 & 693024 & 138378 & 415890 & 554268 & 692646 \\
\hline 9 & 91 & 1 & 154180 & 461680 & 615860 & 770020 & 153760 & 462100 & 615860 & 769600 \\
\hline
\end{tabular}




\begin{tabular}{|c|c|c|c|c|c|c|c|c|c|c|}
\hline & 2 & 2 & 3928 & 11576 & 15504 & 19432 & 3824 & 11680 & 15504 & 19328 \\
\hline 14 & 3 & 2 & 19480 & 58040 & 77520 & 97000 & 19280 & 58240 & 77520 & 96800 \\
\hline 13 & 4 & 2 & 68040 & 203280 & 271320 & 339360 & 67620 & 203700 & 271320 & 338940 \\
\hline 12 & 5 & 2 & 176680 & 528752 & 705432 & 882112 & 176036 & 529396 & 705432 & 881468 \\
\hline 11 & 6 & 2 & 353192 & 1057672 & 1410864 & 1764056 & 352240 & 1058624 & 1410864 & 1763104 \\
\hline 10 & 7 & 2 & 554856 & 1662216 & 217072 & 2771928 & 553680 & 1663392 & 2217072 & 2770752 \\
\hline 9 & & 2 & 693500 & 2077840 & 2771340 & 3464840 & 692170 & 2079170 & 2771340 & 3463510 \\
\hline 13 & 3 & 3 & 90618 & 271152 & 361770 & 452358 & 90282 & 271488 & 361770 & 452022 \\
\hline 12 & 4 & 3 & 294290 & 881440 & 1175730 & 1469990 & 293590 & 882140 & 1175730 & 1469290 \\
\hline 11 & 5 & 3 & 705936 & 2115792 & 2821728 & 3527664 & 704928 & 2116800 & 2821728 & 3526656 \\
\hline 10 & 6 & 3 & 1294012 & 3879176 & 5173188 & 6467140 & 1292612 & 3880576 & 5173188 & 6465740 \\
\hline 9 & 7 & 3 & 1848420 & 5541840 & 7390260 & 9238620 & 1846740 & 5543520 & 7390260 & 9236940 \\
\hline 8 & 8 & 3 & 2079380 & 6234640 & 8314020 & 10393400 & 2077630 & 6236390 & 8314020 & 10391650 \\
\hline 11 & 4 & 4 & 882504 & 2644656 & 3527160 & 4409664 & 881076 & 2646084 & 3527160 & 4408236 \\
\hline 10 & 5 & 4 & 1940904 & 5818848 & 7759752 & 9700656 & 1938972 & 5820780 & 7759752 & 9698724 \\
\hline 9 & 6 & 4 & 3234580 & 9698360 & 12932940 & 16167460 & 231920 & 9701020 & 12932940 & 16164800 \\
\hline 8 & & 4 & 4158480 & 12469560 & 5628040 & 20786520 & 155540 & 12472500 & 6628040 & 20783580 \\
\hline 9 & 5 & 5 & 3881136 & 11638368 & 15519504 & 19400640 & 3878616 & 11640888 & 15519504 & 19398120 \\
\hline 8 & 6 & 5 & 5821424 & 17457832 & 23279256 & 29100680 & 5818204 & 17461052 & 23279256 & 29097460 \\
\hline 7 & 7 & 5 & 6652896 & 19951968 & 26604864 & 33257760 & 6649536 & 19955328 & 26604864 & 33254400 \\
\hline 7 & 6 & 6 & 7761742 & 23277296 & 31039038 & 38800690 & 7757822 & 3281216 & 31039038 & 38796770 \\
\hline 14 & 2 & 2 & 29352 & 86952 & 116304 & 145656 & 28968 & 87288 & 116256 & 145224 \\
\hline 13 & 3 & 2 & 136024 & 406616 & 542640 & 678664 & 135296 & 407344 & 542640 & 677936 \\
\hline 12 & 4 & 2 & 441952 & 1321712 & 1763664 & 2205 & 440468 & 1323028 & 1763 & 2203964 \\
\hline 11 & 5 & 2 & 1059240 & 3173352 & 4232592 & 529 & 57056 & 3175 & 4232 & 5289648 \\
\hline 10 & 6 & 2 & 1942136 & 5817784 & 7759920 & 970 & 000 & 5820584 & 9584 & 9698584 \\
\hline 9 & 7 & 2 & 2773160 & 8312200 & 11085360 & 13858520 & 2769520 & 8315840 & 11085360 & 13854880 \\
\hline 8 & 8 & 2 & 3120540 & 9350700 & 12471240 & 15591780 & 3116550 & 9354270 & 12470820 & 15587370 \\
\hline 12 & 3 & 3 & 588509 & 1762936 & 351445 & 2939939 & 587221 & 1764224 & 2351445 & 2938651 \\
\hline 11 & 4 & 3 & 1764840 & 5289480 & 7054320 & 8819160 & 762320 & 5292000 & 7054320 & 8816640 \\
\hline 10 & 5 & 3 & 3881640 & 1637864 & 15519504 & 19401144 & 378112 & 1641392 & 15519504 & 19397616 \\
\hline 9 & 6 & 3 & 6468850 & 19397000 & 25865850 & 32334670 & 6464090 & 19401760 & 25865850 & 32329910 \\
\hline 8 & 7 & 3 & 8316680 & 24939400 & 33256080 & 41572760 & 8311360 & 24944720 & 33256080 & 41567440 \\
\hline 10 & 4 & 4 & 4853184 & 5448 & & & & 732 & 128 & 7524 \\
\hline 9 & 5 & 4 & 9702840 & 920 & & & & 220 & 3760 & 4849 \\
\hline 8 & 6 & 4 & 214555240 & 3320 & 560 & 800 & 46980 & 5740 & 97720 & 72744700 \\
\hline 7 & 7 & 4 & 216632240 & 49879920 & 66512160 & 83144400 & 6623840 & 49888320 & 66512160 & 83136000 \\
\hline 8 & 5 & 5 & $\begin{array}{ll}2 & 17463432\end{array}$ & 52374336 & 69837768 & 87301200 & 455452 & 52382316 & 69837768 & 87293220 \\
\hline 7 & 6 & 5 & 223284016 & 69833008 & 93117024 & 116401040 & 23274496 & 69842528 & 93117024 & 116391520 \\
\hline 6 & 6 & 6 & 227166848 & 81470256 & 108637104 & 135803904 & 7155646 & 81480336 & 635982 & 135791586 \\
\hline 11 & 3 & 3 & 2352468 & 7053312 & 9405780 & 11758188 & 2350452 & 7055328 & 9405780 & 11756172 \\
\hline 10 & 4 & 3 & 6468432 & 19397448 & 25865880 & 32334192 & 6464568 & 19401312 & 25865880 & 32330328 \\
\hline 9 & 5 & 3 & 312935460 & 38796240 & 51731700 & 64667100 & 12930420 & 38801280 & 51731700 & 64662060 \\
\hline 8 & 6 & 3 & 319 & 94920 & 77597550 & 090 & 190 & 1360 & 77597550 & 96993650 \\
\hline 7 & 7 & 3 & $\begin{array}{ll}3221 \\
\end{array}$ & & & & & 520 & 88682940 & 110850180 \\
\hline 9 & 4 & 4 & & 94880 & 4640 & & & 2020 & 664640 & 80827140 \\
\hline 8 & 5 & 4 & 329103480 & 87292800 & 396280 & 145499760 & 29094660 & 87301620 & 116396280 & 145490940 \\
\hline 7 & & 4 & 338804140 & 116390960 & 195100 & 193999060 & 793500 & 116401600 & 155195100 & 193988420 \\
\hline 7 & 5 & 5 & 346563552 & 139670496 & 186234048 & 232797600 & 6553472 & 139680576 & 186234048 & 232787520 \\
\hline 6 & 6 & 5 & 354324174 & 162948912 & 217273086 & 271597170 & 54312414 & 162960672 & 217273086 & 271585410 \\
\hline 8 & 4 & 4 & 436382500 & 109113480 & 145495980 & 181878480 & 36369900 & 109124820 & 145494720 & 181864620 \\
\hline 7 & & 4 & 458205280 & 174587280 & 232792560 & 290997840 & 58191000 & 174601560 & 232792560 & 290983560 \\
\hline 6 & & 4 & 467909580 & 203682640 & 271592220 & 339501620 & 67892500 & 203698040 & 271590540 & 339482860 \\
\hline 6 & & & 481485376 & 244424208 & & 407394960 & 81469416 & 244440168 & 325909584 & 407379000 \\
\hline 5 & 5 & 5 & 597780440 & 293311068 & 91091496 & 488871936 & 97765320 & 293326188 & 391091496 & 488856816 \\
\hline
\end{tabular}


Table 5 Icosahedral Edge Combinatorics for g irreducible representations up to 4 colors.

\begin{tabular}{|c|c|c|c|c|c|c|c|}
\hline \multicolumn{4}{|c|}{ Partition } & $\mathrm{Ag}_{\mathrm{g}}$ & $\mathrm{T}_{1 \mathrm{~g}}$ & $\mathrm{Gg}_{\mathrm{g}}$ & $\mathrm{Hg}$ \\
\hline 30 & 0 & 0 & 0 & 1 & 0 & 0 & 0 \\
\hline 29 & 1 & 0 & 0 & 1 & 0 & 1 & 2 \\
\hline 28 & 2 & 0 & 0 & 8 & 7 & 15 & 23 \\
\hline 27 & 3 & 0 & 0 & 46 & 91 & 137 & 178 \\
\hline 26 & 4 & 0 & 0 & 262 & 655 & 917 & 1179 \\
\hline 25 & 5 & 0 & 0 & 1257 & 3495 & 4749 & 6006 \\
\hline 24 & 6 & 0 & 0 & 5113 & 14703 & 19816 & 24904 \\
\hline 23 & 7 & 0 & 0 & 17238 & 50622 & 67860 & 85098 \\
\hline 22 & 8 & 0 & 0 & 49270 & 145873 & 195143 & 244413 \\
\hline 21 & 9 & 0 & 0 & 119997 & 356928 & 476925 & 596862 \\
\hline 20 & 10 & 0 & 0 & 251512 & 750094 & 1001597 & 1253109 \\
\hline 19 & 11 & 0 & 0 & 456729 & 1364181 & 1820910 & 2277639 \\
\hline 18 & 12 & 0 & 0 & 722750 & 2160561 & 2883311 & 3605951 \\
\hline 17 & 13 & 0 & 0 & 1000251 & 2991744 & 3991995 & 4992246 \\
\hline 16 & 14 & 0 & 0 & 1214376 & 3633261 & 4847637 & 6062013 \\
\hline 15 & 15 & 0 & 0 & 1295266 & 3875366 & 5170622 & 6465762 \\
\hline 28 & 1 & 1 & 0 & 9 & 20 & 29 & 38 \\
\hline 27 & 2 & 1 & 0 & 113 & 293 & 406 & 519 \\
\hline 26 & 3 & 1 & 0 & 937 & 2717 & 3654 & 4591 \\
\hline 25 & 4 & 1 & 0 & 6019 & 17732 & 23751 & 29770 \\
\hline 24 & 5 & 1 & 0 & 29835 & 88920 & 118755 & 148590 \\
\hline 23 & 6 & 1 & 0 & 119106 & 355914 & 475020 & 594126 \\
\hline 22 & 7 & 1 & 0 & 390754 & 1170026 & 1560780 & 1951534 \\
\hline 21 & 8 & 1 & 0 & 1074073 & 3218072 & 4292145 & 5366218 \\
\hline 20 & 9 & 1 & 0 & 2505217 & 7509788 & 10015005 & 12520222 \\
\hline 19 & 10 & 1 & 0 & 5009719 & 15020291 & 20030010 & 25039729 \\
\hline 18 & 11 & 1 & 0 & 8652111 & 25945179 & 34597290 & 43249401 \\
\hline 17 & 12 & 1 & 0 & 12977523 & 38918412 & 51895935 & 64873458 \\
\hline 16 & 13 & 1 & 0 & 16969947 & 50893968 & 67863915 & 84833862 \\
\hline 15 & 14 & 1 & 0 & 19393980 & 58164780 & 77558760 & 96952740 \\
\hline 26 & 2 & 2 & 0 & 1438 & 4050 & 5488 & 6926 \\
\hline 25 & 3 & 2 & 0 & 12025 & 35477 & 47502 & 59527 \\
\hline 24 & 4 & 2 & 0 & 74698 & 222235 & 296933 & 371631 \\
\hline 23 & 5 & 2 & 0 & 357162 & 1067898 & 1425060 & 1782222 \\
\hline 22 & 6 & 2 & 0 & 1367704 & 4095208 & 5462912 & 6830616 \\
\hline 21 & 7 & 2 & 0 & 4295434 & 12873146 & 17168580 & 21464014 \\
\hline 20 & 8 & 2 & 0 & 11272690 & 33795333 & 45068023 & 56340713 \\
\hline 19 & 9 & 2 & 0 & 25045735 & 75104315 & 100150050 & 125195785 \\
\hline 18 & 10 & 2 & 0 & 47583250 & 142702846 & 190286096 & 237869346 \\
\hline 17 & 11 & 2 & 0 & 77858703 & 233516907 & 311375610 & 389234313 \\
\hline 16 & 12 & 2 & 0 & 110297148 & 330819801 & 441116949 & 551414097 \\
\hline 15 & 13 & 2 & 0 & 135747564 & 407163756 & 542911320 & 678658884 \\
\hline 14 & 14 & 2 & 0 & 145443696 & 436248720 & 581692416 & 727136112 \\
\hline 24 & 3 & 3 & 0 & 99270 & 296595 & 395865 & 495090 \\
\hline 23 & 4 & 3 & 0 & 594750 & 1780350 & 2375100 & 2969850 \\
\hline 22 & 5 & 3 & 0 & 2733042 & 8192418 & 10925460 & 13658502 \\
\hline 21 & 6 & 3 & 0 & 10018926 & 30041154 & 40060080 & 50078826 \\
\hline 20 & 7 & 3 & 0 & 30050878 & 90129182 & 120180060 & 150230938 \\
\hline
\end{tabular}




\begin{tabular}{|c|c|c|c|c|c|c|c|}
\hline 19 & 8 & 3 & 0 & 75122905 & 225327245 & 300450150 & 375573055 \\
\hline 18 & 9 & 3 & 0 & 158584995 & 475698795 & 634283790 & 792868365 \\
\hline 17 & 10 & 3 & 0 & 285447591 & 856262979 & 1141710570 & 1427158161 \\
\hline 16 & 11 & 3 & 0 & 441139257 & 1323322533 & 1764461790 & 2205601047 \\
\hline 15 & 12 & 3 & 0 & 588182454 & 1764433476 & 2352615930 & 2940797754 \\
\hline 14 & 13 & 3 & 0 & 678669180 & 2035887420 & 2714556600 & 3393225780 \\
\hline 22 & 4 & 4 & 0 & 3417258 & 10239840 & 13657098 & 17074356 \\
\hline 21 & 5 & 4 & 0 & 15027870 & 45062160 & 60090030 & 75117900 \\
\hline 20 & 6 & 4 & 0 & 52590538 & 157725568 & 210316106 & 262906644 \\
\hline 19 & 7 & 4 & 0 & 150242950 & 450657350 & 600900300 & 751143250 \\
\hline 18 & 8 & 4 & 0 & 356815030 & 1070325685 & 1427140715 & 1783955745 \\
\hline 17 & 9 & 4 & 0 & 713609325 & 2140667100 & 2854276425 & 3567885750 \\
\hline 16 & 10 & 4 & 0 & 1213123626 & 3639150801 & 4852274427 & 6065398053 \\
\hline 15 & 11 & 4 & 0 & 1764526140 & 5293321020 & 7057847160 & 8822373300 \\
\hline 14 & 12 & 4 & 0 & 2205652956 & 6616662000 & 8822314956 & 11027967912 \\
\hline 13 & 13 & 4 & 0 & 2375312100 & 7125636000 & 9500948100 & 11876260200 \\
\hline 20 & 5 & 5 & 0 & 63103332 & 189274803 & 252378120 & 315481452 \\
\hline 19 & 6 & 5 & 0 & 210334410 & 630926010 & 841260420 & 1051594830 \\
\hline 18 & 7 & 5 & 0 & 570883170 & 1712537970 & 2283421140 & 2854304310 \\
\hline 17 & 8 & 5 & 0 & 1284471045 & 3853226520 & 5137697565 & 6422168610 \\
\hline 16 & 9 & 5 & 0 & 2426194485 & 7278345360 & 9704539845 & 12130734330 \\
\hline 15 & 10 & 5 & 0 & 3881895744 & 11645368026 & 15527263740 & 19409159484 \\
\hline 14 & 11 & 5 & 0 & 5293475460 & 15880066020 & 21173541480 & 26467016940 \\
\hline 13 & 12 & 5 & 0 & 6175715364 & 18526749696 & 24702465060 & 30878180424 \\
\hline 18 & 6 & 6 & 0 & 666038920 & 1997955960 & 2663994880 & 3330033160 \\
\hline 17 & 7 & 6 & 0 & 1712623770 & 5137639650 & 6850263420 & 8562887190 \\
\hline 16 & 8 & 6 & 0 & 3639297090 & 10917520185 & 14556817275 & 18196114365 \\
\hline 15 & 9 & 6 & 0 & 6469809480 & 19408963860 & 25878773340 & 32348581560 \\
\hline 14 & 10 & 6 & 0 & 9704693856 & 29113477536 & 38818171392 & 48522865248 \\
\hline 13 & 11 & 6 & 0 & 12351394692 & 37053535428 & 49404930120 & 61756324812 \\
\hline 12 & 12 & 6 & 0 & 13380683118 & 40141339056 & 53522022174 & 66902703702 \\
\hline 16 & 7 & 7 & 0 & 4159167870 & 12477186150 & 16636354020 & 20795521890 \\
\hline 15 & 8 & 7 & 0 & 8318301420 & 24954406620 & 33272708040 & 41591009460 \\
\hline 14 & 9 & 7 & 0 & 13863778500 & 41590734900 & 55454513400 & 69318291900 \\
\hline 13 & 10 & 7 & 0 & 19409265876 & 58227052884 & 77636318760 & 97045584636 \\
\hline 12 & 11 & 7 & 0 & 22938201468 & 68813811612 & 91752013080 & 114690214548 \\
\hline 14 & 8 & 8 & 0 & 15596775480 & 46789567110 & 62386342590 & 77983118070 \\
\hline 13 & 9 & 8 & 0 & 24261567330 & 72783831120 & 97045398450 & 121306965780 \\
\hline 12 & 10 & 8 & 0 & 31540028520 & 94619010486 & 126159039006 & 157699067526 \\
\hline 11 & 11 & 8 & 0 & 34407266166 & 103220753454 & 137628019620 & 172035285786 \\
\hline 12 & 9 & 9 & 0 & 35044420110 & 105132267240 & 140176687350 & 175221105360 \\
\hline 11 & 10 & 9 & 0 & 42053285274 & 126158738706 & 168212023980 & 210265309254 \\
\hline 10 & 10 & 10 & 0 & 46258637244 & 138774614388 & 185033251584 & 231291888828 \\
\hline 27 & 1 & 1 & 1 & 206 & 606 & 812 & 1018 \\
\hline 26 & 2 & 1 & 1 & 2765 & 8197 & 10962 & 13727 \\
\hline 25 & 3 & 1 & 1 & 23790 & 71214 & 95004 & 118794 \\
\hline 24 & 4 & 1 & 1 & 148603 & 445172 & 593775 & 742378 \\
\hline 23 & 5 & 1 & 1 & 712764 & 2137356 & 2850120 & 3562884 \\
\hline 22 & 6 & 1 & 1 & 2732002 & 8193458 & 10925460 & 13657462 \\
\hline 21 & 7 & 1 & 1 & 8585148 & 25752012 & 34337160 & 42922308 \\
\hline
\end{tabular}




\begin{tabular}{|c|c|c|c|c|c|c|c|}
\hline 20 & 8 & 1 & 1 & 22535513 & 67599532 & 90135045 & 112670558 \\
\hline 19 & 9 & 1 & 1 & 50077170 & 150222930 & 200300100 & 250377270 \\
\hline 18 & 10 & 1 & 1 & 95146051 & 285424139 & 380570190 & 475716241 \\
\hline 17 & 11 & 1 & 1 & 155691666 & 467059554 & 622751220 & 778442886 \\
\hline 16 & 12 & 1 & 1 & 220562979 & 661667916 & 882230895 & 1102793874 \\
\hline 15 & 13 & 1 & 1 & 271460808 & 814361832 & 1085822640 & 1357283448 \\
\hline 14 & 14 & 1 & 1 & 290851356 & 872530044 & 1163381400 & 1454232756 \\
\hline 25 & 2 & 2 & 1 & 35789 & 106717 & 142506 & 178295 \\
\hline 24 & 3 & 2 & 1 & 297193 & 890357 & 1187550 & 1484743 \\
\hline 23 & 4 & 2 & 1 & 1782378 & 5342922 & 7125300 & 8907678 \\
\hline 22 & 5 & 2 & 1 & 8195850 & 24580530 & 32776380 & 40972230 \\
\hline 21 & 6 & 2 & 1 & 30049162 & 90130898 & 120180060 & 150229222 \\
\hline 20 & 7 & 2 & 1 & 90141194 & 270398986 & 360540180 & 450681374 \\
\hline 19 & 8 & 2 & 1 & 225348695 & 676001755 & 901350450 & 1126699145 \\
\hline 18 & 9 & 2 & 1 & 475727395 & 1427123555 & 1902850950 & 2378578345 \\
\hline 17 & 10 & 2 & 1 & 856304163 & 2568827547 & 3425131710 & 4281435873 \\
\hline 16 & 11 & 2 & 1 & 1323371439 & 3970013931 & 5293385370 & 6616756809 \\
\hline 15 & 12 & 2 & 1 & 1764491820 & 5293355340 & 7057847160 & 8822338980 \\
\hline 14 & 13 & 2 & 1 & 2035949196 & 6107720604 & 8143669800 & 10179618996 \\
\hline 23 & 3 & 3 & 1 & 2375568 & 7124832 & 9500400 & 11875968 \\
\hline 22 & 4 & 3 & 1 & 13658658 & 40968642 & 54627300 & 68285958 \\
\hline 21 & 5 & 3 & 1 & 60092604 & 180267516 & 240360120 & 300452724 \\
\hline 20 & 6 & 3 & 1 & 210321826 & 630938594 & 841260420 & 1051582246 \\
\hline 19 & 7 & 3 & 1 & 600908880 & 1802692320 & 2403601200 & 3004510080 \\
\hline 18 & 8 & 3 & 1 & 1427155015 & 4281397835 & 5708552850 & 7135707865 \\
\hline 17 & 9 & 3 & 1 & 2854295730 & 8562809970 & 11417105700 & 14271401430 \\
\hline 16 & 10 & 3 & 1 & 4852300167 & 14556779523 & 19409079690 & 24261379857 \\
\hline 15 & 11 & 3 & 1 & 7057878048 & 21173510592 & 28231388640 & 35289266688 \\
\hline 14 & 12 & 3 & 1 & 8822349276 & 26466886524 & 35289235800 & 44111585076 \\
\hline 13 & 13 & 3 & 1 & 9500984136 & 28502808264 & 38003792400 & 47504776536 \\
\hline 21 & 4 & 4 & 1 & 75118758 & 225331392 & 300450150 & 375568908 \\
\hline 20 & 5 & 4 & 1 & 315482310 & 946408320 & 1261890630 & 1577372940 \\
\hline 19 & 6 & 4 & 1 & 1051597690 & 3154704410 & 4206302100 & 5257899790 \\
\hline 18 & 7 & 4 & 1 & 2854307170 & 8562798530 & 11417105700 & 14271412870 \\
\hline 17 & 8 & 4 & 1 & 6422175045 & 19266312780 & 25688487825 & 32110662870 \\
\hline 16 & 9 & 4 & 1 & 12130740765 & 36391958460 & 48522699225 & 60653439990 \\
\hline 15 & 10 & 4 & 1 & 19409169780 & 58227148980 & 77636318760 & 97045488540 \\
\hline 14 & 11 & 4 & 1 & 26467027236 & 79400680164 & 105867707400 & 132334734636 \\
\hline 13 & 12 & 4 & 1 & 30878192436 & 92634132864 & 123512325300 & 154390517736 \\
\hline 19 & 5 & 5 & 1 & 1261903500 & 3785659020 & 5047562520 & 6309466020 \\
\hline 18 & 6 & 5 & 1 & 3996019170 & 11987928810 & 15983947980 & 19979967150 \\
\hline 17 & 7 & 5 & 1 & 10275433740 & 30826146780 & 41101580520 & 51377014260 \\
\hline 16 & 8 & 5 & 1 & 21835287045 & 65505571560 & 87340858605 & 109176145650 \\
\hline 15 & 9 & 5 & 1 & 38818236600 & 116454400920 & 155272637520 & 194090874120 \\
\hline 14 & 10 & 5 & 1 & 58227354900 & 174681601380 & 232908956280 & 291136311180 \\
\hline 13 & 11 & 5 & 1 & 74107503288 & 222322077432 & 296429580720 & 370537084008 \\
\hline 12 & 12 & 5 & 1 & 80283146580 & 240848899200 & 321132045780 & 401415192360 \\
\hline 17 & 6 & 6 & 1 & 11988031770 & 35963812170 & 47951843940 & 59939875710 \\
\hline 16 & 7 & 6 & 1 & 29113711770 & 87340766370 & 116454478140 & 145568189910 \\
\hline 15 & 8 & 6 & 1 & 58227389220 & 174681567060 & 232908956280 & 291136345500 \\
\hline
\end{tabular}




\begin{tabular}{|c|c|c|c|c|c|c|}
\hline 149 & 6 & 1 & 97045574340 & 291136019460 & 388181593800 & 485227168140 \\
\hline 1310 & 6 & 1 & 135863780052 & 407590451268 & 543454231320 & 679318011372 \\
\hline 1211 & 6 & 1 & 160566257124 & 481697834436 & 642264091560 & 802830348684 \\
\hline 157 & 7 & 1 & 66545519040 & 199636145280 & 266181664320 & 332727183360 \\
\hline 14 & 7 & 1 & 124772839620 & 374317780980 & 499090620600 & 623863460220 \\
\hline 13 & 7 & 1 & 194090977080 & 582272210520 & 776363187600 & 970454164680 \\
\hline 1210 & 7 & 1 & 252318294228 & 756953849652 & 1009272143880 & 1261590438108 \\
\hline 1111 & 7 & 1 & 275256255456 & 825767901504 & 1101024156960 & 1376280412416 \\
\hline 138 & 8 & 1 & 218352424290 & 655056161760 & 873408586050 & 1091761010340 \\
\hline 12 & 8 & 1 & 315397852770 & 946192327080 & 1261590179850 & 1576988032620 \\
\hline 1110 & 8 & 1 & 378477405306 & 1135430810514 & 1513908215820 & 1892385621126 \\
\hline 119 & 9 & 1 & 420530330220 & 1261589909580 & 1682120239800 & 2102650570020 \\
\hline 1010 & 9 & 1 & 462583435314 & 1387748828466 & 1850332263780 & 2312915699094 \\
\hline 242 & 2 & 2 & 446290 & 1335126 & 1781416 & 2227706 \\
\hline 23 & 2 & 2 & 3564600 & 10686000 & 14250600 & 17815200 \\
\hline 22 & 2 & 2 & 20491380 & 61450116 & 81941496 & 102432876 \\
\hline 21 & 2 & 2 & 90145770 & 270394410 & 360540180 & 450685950 \\
\hline 20 & 2 & 2 & 315496324 & 946396308 & 1261892632 & 1577388956 \\
\hline 19 & 2 & 2 & 901386200 & 2704015600 & 3605401800 & 4506788000 \\
\hline 18 & 2 & 2 & 2140768630 & 6422065650 & 8562834280 & 10703602910 \\
\hline 17 & 2 & 2 & 4281495075 & 12844163475 & 17125658550 & 21407153625 \\
\hline 1610 & 2 & 2 & 7278517818 & 21835110726 & 29113628544 & 36392146362 \\
\hline 1511 & 2 & 2 & 10586899440 & 31760183520 & 42347082960 & 52933982400 \\
\hline 1412 & 2 & 2 & 13233615720 & 39700249992 & 52933865712 & 66167481432 \\
\hline 1313 & 2 & 2 & 14251572300 & 42754116300 & 57005688600 & 71257260900 \\
\hline 22 & 3 & 2 & 27317160 & 81937440 & 109254600 & 136571760 \\
\hline 21 & 3 & 2 & 150236658 & 450663642 & 600900300 & 751136958 \\
\hline 20 & 3 & 2 & 630963762 & 1892817498 & 2523781260 & 3154745022 \\
\hline 19 & 3 & 2 & 2103192520 & 6309411680 & 8412604200 & 10515796720 \\
\hline 18 & 3 & 2 & 5708611480 & 17125599920 & 22834211400 & 28542822880 \\
\hline 17 & 3 & 2 & 12844343655 & 38532631995 & 51376975650 & 64221319305 \\
\hline 169 & 3 & 2 & 24261475095 & 72783923355 & 97045398450 & 121306873545 \\
\hline 1510 & 3 & 2 & 38818329264 & 116454308256 & 155272637520 & 194090966784 \\
\hline 1411 & 3 & 2 & 52934044176 & 158801370624 & 211735414800 & 264669458976 \\
\hline 1312 & 3 & 2 & 61756372860 & 185268277740 & 247024650600 & 308781023460 \\
\hline 204 & 4 & 2 & 788717358 & 2366012220 & 3154729578 & 3943446936 \\
\hline 19 & 4 & 2 & 3154784490 & 9464121810 & 12618906300 & 15773690790 \\
\hline 18 & 4 & 2 & 9990091540 & 29969788420 & 39959879960 & 49949971500 \\
\hline 17 & 4 & 2 & 25688661570 & 77065289730 & 102753951300 & 128442612870 \\
\hline 16 & 4 & 2 & 54588323790 & 163763845245 & 218352169035 & 272940492825 \\
\hline 159 & 4 & 2 & 97045745940 & 291135847860 & 388181593800 & 485227339740 \\
\hline 1410 & 4 & 2 & 145568564856 & 436703861880 & 582272426736 & 727840991592 \\
\hline 1311 & 4 & 2 & 185268974436 & 555804977364 & 741073951800 & 926342926236 \\
\hline 1212 & 4 & 2 & 200708076660 & 602122079832 & 802830156492 & 1003538233152 \\
\hline $\begin{array}{ll}18 & 5\end{array}$ & 5 & 2 & 11988048930 & 35963795010 & 47951843940 & 59939892870 \\
\hline 17 & 5 & 2 & 35964069570 & 107891462250 & 143855531820 & 179819601390 \\
\hline 16 & 5 & 2 & 87341109570 & 262022324850 & 349363434420 & 436704543990 \\
\hline 15 & 5 & 2 & 174682116180 & 524044752660 & 698726868840 & 873408985020 \\
\hline 149 & 5 & 2 & 291136671540 & 873408109860 & 1164544781400 & 1455681452940 \\
\hline 1310 & 5 & 2 & 407591268084 & 1222771425876 & 1630362693960 & 2037953962044 \\
\hline
\end{tabular}




\begin{tabular}{|c|c|c|c|c|c|c|}
\hline 1211 & 5 & 2 & 481698699300 & 1445093575380 & 1926792274680 & 2408490973980 \\
\hline 166 & 6 & 2 & 101898053400 & 305692650120 & 407590703520 & 509488756920 \\
\hline 15 & 6 & 2 & 232909453920 & 698726371200 & 931635825120 & 1164545279040 \\
\hline 14 & 6 & 2 & 436705080240 & 1310112151920 & 1746817232160 & 2183522312400 \\
\hline 13 & 6 & 2 & 679318660020 & 2037952496580 & 2717271156600 & 3396589816620 \\
\hline 1210 & 6 & 2 & 883114240008 & 2649338347656 & 3532452587664 & 4415566827672 \\
\hline 1111 & 6 & 2 & 963397182384 & 2890187366976 & 3853584549360 & 4816981731744 \\
\hline 147 & 7 & 2 & 499091255520 & 1497271226880 & 1996362482400 & 2495453737920 \\
\hline 13 & 7 & 2 & 873409516980 & 2620224827220 & 3493634344200 & 4367043861180 \\
\hline 12 & 7 & 2 & 1261591230900 & 3784769488500 & 5046360719400 & 6307951950300 \\
\hline 1110 & 7 & 2 & 1513909405008 & 4541723458272 & 6055632863280 & 7569542268288 \\
\hline 128 & 8 & 2 & 1419290352480 & 4257865561950 & 5677155914430 & 7096446266910 \\
\hline 119 & 8 & 2 & 1892386666170 & 5677154412930 & 7569541079100 & 9461927745270 \\
\hline 1010 & 8 & 2 & 2081625485940 & 6244869827196 & 8326495313136 & 10408120799076 \\
\hline 109 & 9 & 2 & 2312916816210 & 6938744502690 & 9251661318900 & 11564578135110 \\
\hline 21 & 3 & 3 & 200305368 & 600895152 & 801200520 & 1001505528 \\
\hline 20 & 3 & 3 & 1051594830 & 3154707270 & 4206302100 & 5257896930 \\
\hline 19 & 3 & 3 & 4206327840 & 12618880560 & 16825208400 & 21031536240 \\
\hline 18 & 3 & 3 & 13320021420 & 39959805600 & 53279827020 & 66599847180 \\
\hline 17 & 3 & 3 & 34251394320 & 102753874080 & 137005268400 & 171256662720 \\
\hline 16 & 3 & 3 & 72784193625 & 218352001725 & 291136195350 & 363920388975 \\
\hline 159 & 3 & 3 & 129394019880 & 388181439360 & 517575459240 & 646969476600 \\
\hline 1410 & 3 & 3 & 194091028560 & 582272159040 & 776363187600 & 970454216160 \\
\hline 1311 & 3 & 3 & 247024866816 & 741073735584 & 988098602400 & 1235123469216 \\
\hline 1212 & 3 & 3 & 267610309470 & 802829844180 & 1070440153650 & 1338050459970 \\
\hline 194 & 4 & 3 & 5257939830 & 15773570670 & 21031510500 & 26289450330 \\
\hline 18 & 4 & 3 & 19980027210 & 59939712690 & 79919739900 & 99899767110 \\
\hline 17 & 4 & 3 & 59940004410 & 179819215290 & 239759219700 & 299699224110 \\
\hline 16 & 4 & 3 & 145568361510 & 436704029190 & 582272390700 & 727840752210 \\
\hline 15 & 4 & 3 & 291136620060 & 873408161340 & 1164544781400 & 1455681401460 \\
\hline 149 & 4 & 3 & 485227494180 & 1455680474820 & 1940907969000 & 2426135463180 \\
\hline 1310 & 4 & 3 & 679318419780 & 2037952736820 & 2717271156600 & 3396589576380 \\
\hline 1211 & 4 & 3 & 802830781116 & 2408489676684 & 3211320457800 & 4014151238916 \\
\hline $17 \quad 5$ & 5 & 3 & 71927881740 & 215783181900 & 287711063640 & 359638945380 \\
\hline 16 & 5 & 3 & 203795613450 & 611385733530 & 815181346980 & 1018976960430 \\
\hline 15 & 5 & 3 & 465818221440 & 1397453428800 & 1863271650240 & 2329089871680 \\
\hline 14 & 5 & 3 & 873409139460 & 2620225204740 & 3493634344200 & 4367043483660 \\
\hline 139 & 5 & 3 & 1358636118840 & 4075906194360 & 5434542313200 & 6793178432040 \\
\hline 1210 & 5 & 3 & 1766227026564 & 5298677980596 & 7064905007160 & 8831132033724 \\
\hline 1111 & 5 & 3 & 1926792923328 & 5780376175392 & 7707169098720 & 9633962022048 \\
\hline 156 & 6 & 3 & 543454798860 & 1630362127680 & 2173816926540 & 2717271721620 \\
\hline 14 & 6 & 3 & 1164545484960 & 3493633640640 & 4658179125600 & 5822724610560 \\
\hline 13 & 6 & 3 & 2037954418500 & 6113859051300 & 8151813469800 & 10189767888300 \\
\hline 12 & 6 & 3 & 2943711592920 & 8831130087780 & 11774841680700 & 14718553267320 \\
\hline 1110 & 6 & 3 & 3532453836912 & 10597356177408 & 14129810014320 & 17662263851232 \\
\hline 137 & 7 & 3 & 2329090283520 & 6987267967680 & 9316358251200 & 11645448534720 \\
\hline 12 & 7 & 3 & 3784771770780 & 11354310387420 & 15139082158200 & 18923853928980 \\
\hline 119 & 7 & 3 & 5046361800480 & 15139081077120 & 20185442877600 & 25231804678080 \\
\hline 1010 & 7 & 3 & 5550998268816 & 16652988896544 & 22203987165360 & 27754985434176 \\
\hline 118 & 8 & 3 & 5677157475990 & 17031465761310 & 22708623237300 & 28385780713290 \\
\hline
\end{tabular}




\begin{tabular}{|c|c|c|c|c|c|c|c|}
\hline 10 & & 8 & 3 & 6938747745930 & 20816236210770 & 27754983956700 & 34693731702630 \\
\hline 9 & 9 & 9 & 3 & 7709719119900 & 23129151945900 & 30838871065800 & 38548590177300 \\
\hline 18 & 4 & 4 & 4 & 24975105870 & 74924584020 & 99899689890 & 124874795760 \\
\hline 17 & 5 & 4 & 4 & 89909987310 & 269728842240 & 359638829550 & 449548816860 \\
\hline 16 & 6 & 4 & 4 & 254744751690 & 764231977080 & 1018976728770 & 1273721480460 \\
\hline 15 & 7 & 4 & 4 & 582273137160 & 1746816425640 & 2329089562800 & 2911362699960 \\
\hline 14 & 8 & 4 & 4 & 1091761919820 & 3275281100520 & 4367043020340 & 5458804940160 \\
\hline 13 & 9 & 4 & 4 & 1698295779180 & 5094882112320 & 6793177891500 & 8491473670680 \\
\hline 12 & 10 & 4 & 4 & 2207784494916 & 6623346890160 & 8831131385076 & 11038915879992 \\
\hline 11 & 11 & 4 & 4 & 2408491910916 & 7225469462484 & 9633961373400 & 12042453284316 \\
\hline 16 & 5 & 5 & 4 & 305693400870 & 917078619600 & 1222772020470 & 1528465421340 \\
\hline 15 & 6 & 5 & 4 & 815182144920 & 2445543243000 & 3260725387920 & 4075907532840 \\
\hline 14 & 7 & 5 & 4 & 1746818175960 & 5240450512440 & 6987268688400 & 8734086864360 \\
\hline 13 & 8 & 5 & 4 & 3056931537660 & 9170788667040 & 12227720204700 & 15284651742360 \\
\hline 12 & 9 & 5 & 4 & 4415567296140 & 13246695221760 & 17662262517900 & 22077829814040 \\
\hline 11 & 10 & 5 & 4 & 5298680647260 & 15896034374220 & 21194715021480 & 26493395668740 \\
\hline 14 & 6 & 6 & 4 & 2037954959040 & 6113858630880 & 8151813589920 & 10189768548960 \\
\hline 13 & 7 & 6 & 4 & 4075908596760 & 12227718342840 & 16303626939600 & 20379535536360 \\
\hline 12 & & 6 & 4 & 6623351274540 & 19870042712520 & 26493393987060 & 33116745261600 \\
\hline 11 & 9 & 6 & 4 & 8831134051740 & 26493390984060 & 35324525035800 & 44155659087540 \\
\hline 10 & 10 & 6 & 4 & 9714247799256 & 29142729992376 & 38856977791632 & 48571225590888 \\
\hline 12 & 7 & 7 & 4 & 7569543301320 & 22708621015080 & 30278164316400 & 37847707617720 \\
\hline 11 & 8 & 7 & 4 & 11354314591620 & 34062931882980 & 45417246474600 & 56771561066220 \\
\hline 10 & 9 & 7 & 4 & 13877495131500 & 41632472781900 & 55509967913400 & 69387463044900 \\
\hline 10 & 8 & 8 & 4 & 15612182766180 & 46836531451710 & 62448714217890 & 78060896984070 \\
\hline 9 & 9 & 8 & 4 & 17346868689150 & 52040591202600 & 69387459891750 & 86734328580900 \\
\hline 15 & 5 & 5 & 5 & 978218079720 & 2934652385820 & 3912870465480 & 4891088545200 \\
\hline 14 & 6 & 5 & 5 & 2445545096280 & 7336631067480 & 9782176163760 & 12227721260040 \\
\hline 13 & 7 & 5 & 5 & 4891089162960 & 14673263164560 & 19564352327520 & 24455441490480 \\
\hline 12 & 8 & 5 & 5 & 7948019979900 & 23844052552320 & 31792072532220 & 39740092512120 \\
\hline 11 & 9 & 5 & 5 & 10597359132360 & 31792070910600 & 42389430042960 & 52986789175320 \\
\hline 10 & 10 & 5 & 5 & 11657095478064 & 34971277569246 & 46628373047220 & 58285468525284 \\
\hline 13 & 6 & 6 & 5 & 5706271410840 & 17118806304600 & 22825077715440 & 28531349126280 \\
\hline 12 & 7 & 6 & 5 & 10597359853080 & 31792070189880 & 42389430042960 & 52986789896040 \\
\hline 11 & 8 & 6 & 5 & 15896039419260 & 47688105645180 & 63584145064440 & 79480184483700 \\
\hline 10 & 9 & 6 & 5 & 19428492103020 & 58285462975740 & 77713955078760 & 97142447181780 \\
\hline 11 & 7 & 7 & 5 & 18166900752000 & 54500693607360 & 72667594359360 & 90834495111360 \\
\hline 10 & 8 & 7 & 5 & 24979489074540 & 74938453169580 & 99917942244120 & 124897431318660 \\
\hline 9 & 9 & 7 & 5 & 27754986659400 & 83264949167400 & 111019935826800 & 138774922486200 \\
\hline 9 & 8 & 8 & 5 & 31224361117950 & 93673066687200 & 124897427805150 & 156121788923100 \\
\hline 12 & 6 & 6 & 6 & 12363587559480 & 37090747774080 & 49454335333560 & 61817922883560 \\
\hline 11 & 7 & 6 & 6 & 21194718985440 & 63584141100480 & 84778860085920 & 105973579071360 \\
\hline 10 & 8 & 6 & 6 & 29142738905280 & 87428194133280 & 116570933038560 & 145713671943840 \\
\hline 9 & 9 & 6 & 6 & 32380819575300 & 97142438893500 & 129523258468800 & 161904078031500 \\
\hline 10 & 7 & 7 & 6 & 33305985192480 & 99917937799680 & 133223922992160 & 166529908184640 \\
\hline 9 & 8 & 7 & 6 & 41632481190300 & 124897422549900 & 166529903740200 & 208162384930500 \\
\hline 8 & 8 & 8 & 6 & 46836542652900 & 140509599580350 & 187346142233250 & 234182684886150 \\
\hline 9 & 7 & 7 & 7 & 47579976100800 & 142739913888000 & 190319889988800 & 237899866089600 \\
\hline 8 & 8 & 7 & 7 & 53527474614900 & 160582401622500 & 214109876237400 & 267637350852300 \\
\hline
\end{tabular}


Table 6 Icosahedral Edge Combinatorics of u irreducible representations (4 colors)

\begin{tabular}{|c|c|c|c|c|c|c|c|}
\hline \multicolumn{4}{|c|}{ Partiti on } & $A_{u}$ & $\mathrm{~T}_{1 \mathrm{u}}$ & $\mathrm{Gu}_{\mathrm{u}}$ & $\mathrm{H}_{\mathrm{u}}$ \\
\hline 30 & 0 & 0 & 0 & 0 & 0 & 0 & 0 \\
\hline 29 & 1 & 0 & 0 & 0 & 1 & 1 & 1 \\
\hline 28 & 2 & 0 & 0 & 3 & 11 & 14 & 17 \\
\hline 27 & 3 & 0 & 0 & 32 & 105 & 137 & 164 \\
\hline 26 & 4 & 0 & 0 & 221 & 689 & 910 & 1131 \\
\hline 25 & 5 & 0 & 0 & 1166 & 3586 & 4749 & 5915 \\
\hline 24 & 6 & 0 & 0 & 4912 & 14872 & 19784 & 24676 \\
\hline 23 & 7 & 0 & 0 & 16874 & 50986 & 67860 & 84734 \\
\hline 22 & 8 & 0 & 0 & 48620 & 146432 & 195052 & 243672 \\
\hline 21 & 9 & 0 & 0 & 118996 & 357929 & 476925 & 595861 \\
\hline 201 & 10 & 0 & 0 & 249995 & 751409 & 1001398 & 1251393 \\
\hline 191 & 11 & 0 & 0 & 454727 & 1366183 & 1820910 & 2275637 \\
\hline 181 & 12 & 0 & 0 & 720125 & 2162849 & 2882974 & 3602999 \\
\hline 171 & 13 & 0 & 0 & 997248 & 2994747 & 3991995 & 4989243 \\
\hline 161 & 14 & 0 & 0 & 1210944 & 3636264 & 4847208 & 6058152 \\
\hline 151 & 15 & 0 & 0 & 1291834 & 3878798 & 5170622 & 6462330 \\
\hline 28 & 1 & 1 & 0 & 6 & 23 & 29 & 35 \\
\hline 27 & 2 & 1 & 0 & 97 & 309 & 406 & 503 \\
\hline 26 & 3 & 1 & 0 & 897 & 2757 & 3654 & 4551 \\
\hline 25 & 4 & 1 & 0 & 5902 & 17849 & 23751 & 29653 \\
\hline 24 & 5 & 1 & 0 & 29588 & 89167 & 118755 & 148343 \\
\hline 23 & 6 & 1 & 0 & 118586 & 356434 & 475020 & 593606 \\
\hline 22 & 7 & 1 & 0 & 389818 & 1170962 & 1560780 & 1950598 \\
\hline 21 & 8 & 1 & 0 & 1072500 & 3219645 & 4292145 & 5364645 \\
\hline 20 & 9 & 1 & 0 & 2502786 & 7512219 & 10015005 & 12517791 \\
\hline 191 & 10 & 1 & 0 & 5006287 & 15023723 & 20030010 & 25036297 \\
\hline 181 & 11 & 1 & 0 & 8647535 & 25949755 & 34597290 & 43244825 \\
\hline 171 & 12 & 1 & 0 & 12971946 & 38923989 & 51895935 & 64867881 \\
\hline 161 & 13 & 1 & 0 & 16963512 & 50900403 & 67863915 & 84827427 \\
\hline 151 & 14 & 1 & 0 & 19387116 & 58171644 & 77558760 & 96945876 \\
\hline 26 & 2 & 2 & 0 & 1355 & 4119 & 5474 & 6829 \\
\hline 25 & 3 & 2 & 0 & 11817 & 35685 & 47502 & 59319 \\
\hline 24 & 4 & 2 & 0 & 74087 & 222755 & 296842 & 370929 \\
\hline 23 & 5 & 2 & 0 & 355914 & 1069146 & 1425060 & 1780974 \\
\hline 22 & 6 & 2 & 0 & 1365026 & 4097522 & 5462548 & 6827574 \\
\hline 21 & 7 & 2 & 0 & 4290858 & 12877722 & 17168580 & 21459438 \\
\hline 20 & 8 & 2 & 0 & 11264825 & 33802197 & 45067022 & 56331847 \\
\hline 19 & 9 & 2 & 0 & 25034295 & 75115755 & 100150050 & 125184345 \\
\hline 181 & 10 & 2 & 0 & 47566805 & 142717289 & 190284094 & 237850899 \\
\hline 171 & 11 & 2 & 0 & 77838111 & 233537499 & 311375610 & 389213721 \\
\hline 161 & 12 & 2 & 0 & 110271837 & 330842109 & 441113946 & 551385783 \\
\hline 151 & 13 & 2 & 0 & 135720108 & 407191212 & 542911320 & 678631428 \\
\hline 141 & 14 & 2 & 0 & 145414524 & 436274460 & 581688984 & 727103508 \\
\hline 24 & 3 & 3 & 0 & 98776 & 297089 & 395865 & 494596 \\
\hline 23 & 4 & 3 & 0 & 593346 & 1781754 & 2375100 & 2968446 \\
\hline 22 & 5 & 3 & 0 & 2730234 & 8195226 & 10925460 & 13655694 \\
\hline 21 & 6 & 3 & 0 & 10013206 & 30046874 & 40060080 & 50073106 \\
\hline
\end{tabular}




\begin{tabular}{|c|c|c|c|c|c|c|c|}
\hline 20 & 7 & 3 & 0 & 30041154 & 90138906 & 120180060 & 150221214 \\
\hline 19 & 8 & 3 & 0 & 75107175 & 225342975 & 300450150 & 375557325 \\
\hline 18 & 9 & 3 & 0 & 158562115 & 475721675 & 634283790 & 792845485 \\
\hline 17 & 10 & 3 & 0 & 285416703 & 856293867 & 1141710570 & 1427127273 \\
\hline 16 & 11 & 3 & 0 & 441100647 & 1323361143 & 1764461790 & 2205562437 \\
\hline 15 & 12 & 3 & 0 & 588137838 & 1764478092 & 2352615930 & 2940753138 \\
\hline 14 & 13 & 3 & 0 & 678621132 & 2035935468 & 2714556600 & 3393177732 \\
\hline 22 & 4 & 4 & 0 & 3413202 & 10243350 & 13656552 & 17069754 \\
\hline 21 & 5 & 4 & 0 & 15020148 & 45069882 & 60090030 & 75110178 \\
\hline 20 & 6 & 4 & 0 & 52574522 & 157739582 & 210314104 & 262888626 \\
\hline 19 & 7 & 4 & 0 & 150217210 & 450683090 & 600900300 & 751117510 \\
\hline 18 & 8 & 4 & 0 & 356772845 & 1070362865 & 1427135710 & 1783908555 \\
\hline 17 & 9 & 4 & 0 & 713551410 & 2140725015 & 2854276425 & 3567827835 \\
\hline 16 & 10 & 4 & 0 & 1213045119 & 3639220299 & 4852265418 & 6065310537 \\
\hline 15 & 11 & 4 & 0 & 1764433476 & 5293413684 & 7057847160 & 8822280636 \\
\hline 14 & 12 & 4 & 0 & 2205546564 & 6616756380 & 8822302944 & 11027849508 \\
\hline 13 & 13 & 4 & 0 & 2375203992 & 7125744108 & 9500948100 & 11876152092 \\
\hline 20 & 5 & 5 & 0 & 63088746 & 189289389 & 252378120 & 315466866 \\
\hline 19 & 6 & 5 & 0 & 210305810 & 630954610 & 841260420 & 1051566230 \\
\hline 18 & 7 & 5 & 0 & 570837410 & 1712583730 & 2283421140 & 2854258550 \\
\hline 17 & 8 & 5 & 0 & 1284400260 & 3853297305 & 5137697565 & 6422097825 \\
\hline 16 & 9 & 5 & 0 & 2426097960 & 7278441885 & 9704539845 & 12130637805 \\
\hline 15 & 10 & 5 & 0 & 3881772192 & 11645491578 & 15527263740 & 19409035932 \\
\hline 14 & 11 & 5 & 0 & 5293331316 & 15880210164 & 21173541480 & 26466872796 \\
\hline 13 & 12 & 5 & 0 & 6175559208 & 18526905852 & 24702465060 & 30878024268 \\
\hline 18 & 6 & 6 & 0 & 665982190 & 1998006010 & 2663988200 & 3329969770 \\
\hline 17 & 7 & 6 & 0 & 1712537970 & 5137725450 & 6850263420 & 8562801390 \\
\hline 16 & 8 & 6 & 0 & 3639164100 & 10917638160 & 14556802260 & 18195966360 \\
\hline 15 & 9 & 6 & 0 & 6469637880 & 19409135460 & 25878773340 & 32348409960 \\
\hline 14 & 10 & 6 & 0 & 9704475924 & 29113671444 & 38818147368 & 48522623292 \\
\hline 13 & 11 & 6 & 0 & 12351154452 & 37053775668 & 49404930120 & 61756084572 \\
\hline 12 & 12 & 6 & 0 & 13380426852 & 40141567284 & 53521994136 & 66902419428 \\
\hline 16 & 7 & 7 & 0 & 4159039170 & 12477314850 & 16636354020 & 20795393190 \\
\hline 15 & 8 & 7 & 0 & 8318112660 & 24954595380 & 33272708040 & 41590820700 \\
\hline 14 & 9 & 7 & 0 & 13863538260 & 41590975140 & 55454513400 & 69318051660 \\
\hline 13 & 10 & 7 & 0 & 19408977588 & 58227341172 & 77636318760 & 97045296348 \\
\hline 12 & 11 & 7 & 0 & 22937889156 & 68814123924 & 91752013080 & 114689902236 \\
\hline 14 & 8 & 8 & 0 & 15596500920 & 46789811640 & 62386312560 & 77982813480 \\
\hline 13 & 9 & 8 & 0 & 24261237000 & 72784161450 & 97045398450 & 121306635450 \\
\hline 12 & 10 & 8 & 0 & 31539638130 & 94619358834 & 126158996964 & 157698635094 \\
\hline 11 & 11 & 8 & 0 & 34406869770 & 103221149850 & 137628019620 & 172034889390 \\
\hline 12 & 9 & 9 & 0 & 35044029720 & 105132657630 & 140176687350 & 175220714970 \\
\hline 11 & 10 & 9 & 0 & 42052852842 & 126159171138 & 168212023980 & 210264876822 \\
\hline 10 & 10 & 10 & 0 & 46258165170 & 138775036008 & 185033201136 & 231291366306 \\
\hline 27 & 1 & 1 & 1 & 200 & 612 & 812 & 1012 \\
\hline 26 & 2 & 1 & 1 & 2723 & 8239 & 10962 & 13685 \\
\hline 25 & 3 & 1 & 1 & 23712 & 71292 & 95004 & 118716 \\
\hline 24 & 4 & 1 & 1 & 148330 & 445445 & 593775 & 742105 \\
\hline 23 & 5 & 1 & 1 & 712296 & 2137824 & 2850120 & 3562416 \\
\hline 22 & 6 & 1 & 1 & 2730910 & 8194550 & 10925460 & 13656370 \\
\hline
\end{tabular}




\begin{tabular}{|c|c|c|c|c|c|c|c|}
\hline 21 & 7 & 1 & 1 & 8583432 & 25753728 & 34337160 & 42920592 \\
\hline 20 & 8 & 1 & 1 & 22532510 & 67602535 & 90135045 & 112667555 \\
\hline 19 & 9 & 1 & 1 & 50072880 & 150227220 & 200300100 & 250372980 \\
\hline 18 & 10 & 1 & 1 & 95140045 & 285430145 & 380570190 & 475710235 \\
\hline 17 & 11 & 1 & 1 & 155683944 & 467067276 & 622751220 & 778435164 \\
\hline 16 & 12 & 1 & 1 & 220553970 & 661676925 & 882230895 & 1102784865 \\
\hline 15 & 13 & 1 & 1 & 271450512 & 814372128 & 1085822640 & 1357273152 \\
\hline 14 & 14 & 1 & 1 & 290841060 & 872540340 & 1163381400 & 1454222460 \\
\hline 25 & 2 & 2 & 1 & 35555 & 106951 & 142506 & 178061 \\
\hline 24 & 3 & 2 & 1 & 296673 & 890877 & 1187550 & 1484223 \\
\hline 23 & 4 & 2 & 1 & 1780818 & 5344482 & 7125300 & 8906118 \\
\hline 22 & 5 & 2 & 1 & 8192886 & 24583494 & 32776380 & 40969266 \\
\hline 21 & 6 & 2 & 1 & 30042870 & 90137190 & 120180060 & 150222930 \\
\hline 20 & 7 & 2 & 1 & 90130898 & 270409282 & 360540180 & 450671078 \\
\hline 19 & 8 & 2 & 1 & 225331535 & 676018915 & 901350450 & 1126681985 \\
\hline 18 & 9 & 2 & 1 & 475703085 & 1427147865 & 1902850950 & 2378554035 \\
\hline 17 & 10 & 2 & 1 & 856270701 & 2568861009 & 3425131710 & 4281402411 \\
\hline 16 & 11 & 2 & 1 & 1323330255 & 3970055115 & 5293385370 & 6616715625 \\
\hline 15 & 12 & 2 & 1 & 1764443772 & 5293403388 & 7057847160 & 8822290932 \\
\hline 14 & 13 & 2 & 1 & 2035897716 & 6107772084 & 8143669800 & 10179567516 \\
\hline 23 & 3 & 3 & 1 & 2374632 & 7125768 & 9500400 & 11875032 \\
\hline 22 & 4 & 3 & 1 & 13655538 & 40971762 & 54627300 & 68282838 \\
\hline 21 & 5 & 3 & 1 & 60087456 & 180272664 & 240360120 & 300447576 \\
\hline 20 & 6 & 3 & 1 & 210310386 & 630950034 & 841260420 & 1051570806 \\
\hline 19 & 7 & 3 & 1 & 600891720 & 1802709480 & 2403601200 & 3004492920 \\
\hline 18 & 8 & 3 & 1 & 1427126415 & 4281426435 & 5708552850 & 7135679265 \\
\hline 17 & 9 & 3 & 1 & 2854257120 & 8562848580 & 11417105700 & 14271362820 \\
\hline 16 & 10 & 3 & 1 & 4852248687 & 14556831003 & 19409079690 & 24261328377 \\
\hline 15 & 11 & 3 & 1 & 7057816272 & 21173572368 & 28231388640 & 35289204912 \\
\hline 14 & 12 & 3 & 1 & 8822280636 & 26466955164 & 35289235800 & 44111516436 \\
\hline 13 & 13 & 3 & 1 & 9500912064 & 28502880336 & 38003792400 & 47504704464 \\
\hline 21 & 4 & 4 & 1 & 75109320 & 225340830 & 300450150 & 375559470 \\
\hline 20 & 5 & 4 & 1 & 315466008 & 946424622 & 1261890630 & 1577356638 \\
\hline 19 & 6 & 4 & 1 & 1051563370 & 3154738730 & 4206302100 & 5257865470 \\
\hline 18 & 7 & 4 & 1 & 2854255690 & 8562850010 & 11417105700 & 14271361390 \\
\hline 17 & 8 & 4 & 1 & 6422091390 & 19266396435 & 25688487825 & 32110579215 \\
\hline 16 & 9 & 4 & 1 & 12130631370 & 36392067855 & 48522699225 & 60653330595 \\
\hline 15 & 10 & 4 & 1 & 19409025636 & 58227293124 & 77636318760 & 97045344396 \\
\hline 14 & 11 & 4 & 1 & 26466862500 & 79400844900 & 105867707400 & 132334569900 \\
\hline 13 & 12 & 4 & 1 & 30878012256 & 92634313044 & 123512325300 & 154390337556 \\
\hline 19 & 5 & 5 & 1 & 1261877760 & 3785684760 & 5047562520 & 6309440280 \\
\hline 18 & 6 & 5 & 1 & 3995964830 & 11987983150 & 15983947980 & 19979912810 \\
\hline 17 & 7 & 5 & 1 & 10275356520 & 30826224000 & 41101580520 & 51376937040 \\
\hline 16 & 8 & 5 & 1 & 21835164780 & 65505693825 & 87340858605 & 109176023385 \\
\hline 15 & 9 & 5 & 1 & 38818082160 & 116454555360 & 155272637520 & 194090719680 \\
\hline 14 & 10 & 5 & 1 & 58227159276 & 174681797004 & 232908956280 & 291136115556 \\
\hline 13 & 11 & 5 & 1 & 74107287072 & 222322293648 & 296429580720 & 370536867792 \\
\hline 12 & 12 & 5 & 1 & 80282918352 & 240849127428 & 321132045780 & 401414964132 \\
\hline 17 & 6 & 6 & 1 & 11987920230 & 35963923710 & 47951843940 & 59939764170 \\
\hline 16 & 7 & 6 & 1 & 29113557330 & 87340920810 & 116454478140 & 145568035470 \\
\hline
\end{tabular}




\begin{tabular}{|c|c|c|c|c|c|c|c|}
\hline 15 & 8 & 6 & 1 & 58227148980 & 174681807300 & 232908956280 & 291136105260 \\
\hline 14 & 9 & 6 & 1 & 97045282620 & 291136311180 & 388181593800 & 485226876420 \\
\hline 13 & 10 & 6 & 1 & 135863419692 & 407590811628 & 543454231320 & 679317651012 \\
\hline 12 & 11 & 6 & 1 & 160565872740 & 481698218820 & 642264091560 & 802829964300 \\
\hline 15 & 7 & 7 & 1 & 66545313120 & 199636351200 & 266181664320 & 332726977440 \\
\hline 14 & 8 & 7 & 1 & 124772530740 & 374318089860 & 499090620600 & 623863151340 \\
\hline 13 & 9 & 7 & 1 & 194090616720 & 582272570880 & 776363187600 & 970453804320 \\
\hline 12 & 10 & 7 & 1 & 252317861796 & 756954282084 & 1009272143880 & 1261590005676 \\
\hline 11 & 11 & 7 & 1 & 275255823024 & 825768333936 & 1101024156960 & 1376279979984 \\
\hline 13 & 8 & 8 & 1 & 218351973840 & 655056612210 & 873408586050 & 1091760559890 \\
\hline 12 & 9 & 8 & 1 & 315397342260 & 946192837590 & 1261590179850 & 1576987522110 \\
\hline 11 & 10 & 8 & 1 & 378476828730 & 1135431387090 & 1513908215820 & 1892385044550 \\
\hline 11 & 9 & 9 & 1 & 420529789680 & 1261590450120 & 1682120239800 & 2102650029480 \\
\hline 10 & 10 & 9 & 1 & 462582822702 & 1387749441078 & 1850332263780 & 2312915086482 \\
\hline 24 & 2 & 2 & 2 & 445055 & 1336179 & 1781234 & 2226289 \\
\hline 23 & 3 & 2 & 2 & 3561792 & 10688808 & 14250600 & 17812392 \\
\hline 22 & 4 & 2 & 2 & 20483190 & 61457214 & 81940404 & 102423594 \\
\hline 21 & 5 & 2 & 2 & 90130326 & 270409854 & 360540180 & 450670506 \\
\hline 20 & 6 & 2 & 2 & 315464006 & 946424622 & 1261888628 & 1577352634 \\
\hline 19 & 7 & 2 & 2 & 901334720 & 2704067080 & 3605401800 & 4506736520 \\
\hline 18 & 8 & 2 & 2 & 2140683545 & 6422140725 & 8562824270 & 10703507815 \\
\hline 17 & 9 & 2 & 2 & 4281379245 & 12844279305 & 17125658550 & 21407037795 \\
\hline 16 & 10 & 2 & 2 & 7278359517 & 21835251009 & 29113610526 & 36391970043 \\
\hline 15 & 11 & 2 & 2 & 10586714112 & 31760368848 & 42347082960 & 52933797072 \\
\hline 14 & 12 & 2 & 2 & 13233401220 & 39700440468 & 52933841688 & 66167242908 \\
\hline 13 & 13 & 2 & 2 & 14251356084 & 42754332516 & 57005688600 & 71257044684 \\
\hline 22 & 3 & 3 & 2 & 27311232 & 81943368 & 109254600 & 136565832 \\
\hline 21 & 4 & 3 & 2 & 150219498 & 450680802 & 600900300 & 751119798 \\
\hline 20 & 5 & 3 & 2 & 630932874 & 1892848386 & 2523781260 & 3154714134 \\
\hline 19 & 6 & 3 & 2 & 2103129600 & 6309474600 & 8412604200 & 10515733800 \\
\hline 18 & 7 & 3 & 2 & 5708514240 & 17125697160 & 22834211400 & 28542725640 \\
\hline 17 & 8 & 3 & 2 & 12844189215 & 38532786435 & 51376975650 & 64221164865 \\
\hline 16 & 9 & 3 & 2 & 24261269175 & 72784129275 & 97045398450 & 121306667625 \\
\hline 15 & 10 & 3 & 2 & 38818061568 & 116454575952 & 155272637520 & 194090699088 \\
\hline 14 & 11 & 3 & 2 & 52933735296 & 158801679504 & 211735414800 & 264669150096 \\
\hline 13 & 12 & 3 & 2 & 61756036524 & 185268614076 & 247024650600 & 308780687124 \\
\hline 20 & 4 & 4 & 2 & 788668452 & 2366055120 & 3154723572 & 3943392024 \\
\hline 19 & 5 & 4 & 2 & 3154698690 & 9464207610 & 12618906300 & 15773604990 \\
\hline 18 & 6 & 4 & 2 & 9989918510 & 29969941430 & 39959859940 & 49949778450 \\
\hline 17 & 7 & 4 & 2 & 25688404170 & 77065547130 & 102753951300 & 128442355470 \\
\hline 16 & 8 & 4 & 2 & 54587918385 & 163764205605 & 218352123990 & 272940042375 \\
\hline 15 & 9 & 4 & 2 & 97045231140 & 291136362660 & 388181593800 & 485226824940 \\
\hline 14 & 10 & 4 & 2 & 145567900764 & 436704453900 & 582272354664 & 727840255428 \\
\hline 13 & 11 & 4 & 2 & 185268253716 & 555805698084 & 741073951800 & 926342205516 \\
\hline 12 & 12 & 4 & 2 & 200707295880 & 602122776528 & 802830072408 & 1003537368288 \\
\hline 18 & 5 & 5 & 2 & 11987903070 & 35963940870 & 47951843940 & 59939747010 \\
\hline 17 & 6 & 5 & 2 & 35963786430 & 107891745390 & 143855531820 & 179819318250 \\
\hline 16 & 7 & 5 & 2 & 87340697730 & 262022736690 & 349363434420 & 436704132150 \\
\hline 15 & 8 & 5 & 2 & 174681498420 & 524045370420 & 698726868840 & 873408367260 \\
\hline 14 & 9 & 5 & 2 & 291135899340 & 873408882060 & 1164544781400 & 1455680680740 \\
\hline
\end{tabular}




\begin{tabular}{|c|c|c|c|c|c|c|}
\hline 1310 & 5 & 2 & 407590331148 & 1222772362812 & 1630362693960 & 2037953025108 \\
\hline 1211 & 5 & 2 & 481697690292 & 1445094584388 & 1926792274680 & 2408489964972 \\
\hline 16 & 6 & 2 & 101897508570 & 305693134890 & 407590643460 & 509488152030 \\
\hline 15 & 6 & & 232908698880 & 698727126240 & 931635825120 & 1164544524000 \\
\hline 14 & 6 & 2 & 436703956260 & 1310113155780 & 1746817112040 & 2183521068300 \\
\hline 13 & 6 & 2 & 679317338700 & 2037953817900 & 2717271156600 & 3396588495300 \\
\hline 1210 & 6 & 2 & 883112642412 & 2649339777084 & 3532452419496 & 4415565061908 \\
\hline 11 & 6 & 2 & 963395596800 & 2890188952560 & 3853584549360 & 4816980146160 \\
\hline 14 & 7 & 2 & 499090225920 & 1497272256480 & 1996362482400 & 2495452708320 \\
\hline 13 & 7 & 2 & 873408075540 & 2620226268660 & 3493634344200 & 4367042419740 \\
\hline 12 & 7 & 2 & 1261589549220 & 3784771170180 & 5046360719400 & 6307950268620 \\
\hline 1110 & 7 & 2 & 1513907531136 & 4541725332144 & 6055632863280 & 7569540394416 \\
\hline 128 & 8 & 2 & 1419288340470 & 4257867363750 & 5677155704220 & 7096444044690 \\
\hline 11 & 8 & 2 & 1892384504010 & 5677156575090 & 7569541079100 & 9461925583110 \\
\hline 1010 & 8 & 2 & 2081623053510 & 6244872007374 & 8326495060884 & 10408118114394 \\
\hline 109 & 9 & 2 & 2312914473870 & 6938746845030 & 9251661318900 & 11564575792770 \\
\hline 21 & 3 & 3 & 200295072 & 600905448 & 801200520 & 1001495232 \\
\hline 20 & 3 & 3 & 1051562226 & 3154739874 & 4206302100 & 5257864326 \\
\hline 19 & 3 & 3 & 4206276360 & 12618932040 & 16825208400 & 21031484760 \\
\hline 18 & 3 & 3 & 13319912740 & 39959914280 & 53279827020 & 66599738500 \\
\hline 17 & 3 & 3 & 34251239880 & 102754028520 & 137005268400 & 171256508280 \\
\hline 16 & 3 & 3 & 72783949095 & 218352246255 & 291136195350 & 363920144445 \\
\hline 15 & 3 & 3 & 129393711000 & 388181748240 & 517575459240 & 646969167720 \\
\hline 1410 & 3 & 3 & 194090637312 & 582272550288 & 776363187600 & 970453824912 \\
\hline 1311 & 3 & 3 & 247024434384 & 741074168016 & 988098602400 & 1235123036784 \\
\hline 1212 & 3 & 3 & 267609853014 & 802830300636 & 1070440153650 & 1338050003514 \\
\hline 19 & 4 & 3 & 5257845450 & 15773665050 & 21031510500 & 26289355950 \\
\hline 18 & 4 & 3 & 19979872770 & 59939867130 & 79919739900 & 99899612670 \\
\hline 17 & 4 & 3 & 59939695530 & 179819524170 & 239759219700 & 299698915230 \\
\hline 16 & 4 & 3 & 145567923930 & 436704466770 & 582272390700 & 727840314630 \\
\hline 15 & 4 & 3 & 291135950820 & 873408830580 & 1164544781400 & 1455680732220 \\
\hline 14 & 4 & 3 & 485226670500 & 1455681298500 & 1940907969000 & 2426134639500 \\
\hline 1310 & 4 & 3 & 679317410772 & 2037953745828 & 2717271156600 & 3396588567372 \\
\hline 1211 & 4 & 3 & 802829700036 & 2408490757764 & 3211320457800 & 4014150157836 \\
\hline 17 & 5 & 3 & 71927650080 & 215783413560 & 287711063640 & 359638713720 \\
\hline 16 & 5 & 3 & 203795150130 & 611386196850 & 815181346980 & 1018976497110 \\
\hline 15 & 5 & 3 & 465817603680 & 1397454046560 & 1863271650240 & 2329089253920 \\
\hline 14 & 5 & 3 & 873408212820 & 2620226131380 & 3493634344200 & 4367042557020 \\
\hline 13 & 5 & 3 & 1358635037760 & 4075907275440 & 5434542313200 & 6793177350960 \\
\hline 1210 & 5 & 3 & 1766225729268 & 5298679277892 & 7064905007160 & 8831130736428 \\
\hline 1111 & 5 & 3 & 1926791626032 & 5780377472688 & 7707169098720 & 9633960724752 \\
\hline 15 & 6 & 3 & 543453906540 & 1630363020000 & 2173816926540 & 2717270829300 \\
\hline 14 & 6 & 3 & 1164544318080 & 3493634807520 & 4658179125600 & 5822723443680 \\
\hline 13 & 6 & 3 & 2037952736820 & 6113860732980 & 8151813469800 & 10189766206620 \\
\hline 129 & 6 & 3 & 2943709671000 & 8831132009700 & 11774841680700 & 14718551345400 \\
\hline 1110 & 6 & 3 & 3532451674752 & 10597358339568 & 14129810014320 & 17662261689072 \\
\hline 13 & 7 & 3 & 2329088842080 & 6987269409120 & 9316358251200 & 11645447093280 \\
\hline 12 & 7 & 3 & 3784769728740 & 11354312429460 & 15139082158200 & 18923851886940 \\
\hline 11 & 7 & 3 & 5046359638320 & 15139083239280 & 20185442877600 & 25231802515920 \\
\hline 1010 & 7 & 3 & 5550995818368 & 16652991346992 & 22203987165360 & 27754982983728 \\
\hline
\end{tabular}




\begin{tabular}{|c|c|c|c|c|c|c|c|}
\hline 11 & 8 & 8 & & 5677154773290 & 17031468464010 & 22708623237300 & \\
\hline 10 & 9 & 8 & 3 & 6938744863050 & 20816239093650 & 27754983956700 & 34693728819750 \\
\hline 9 & 9 & 9 & 3 & 7709716417200 & 23129154648600 & 30838871065800 & 38548587474600 \\
\hline 18 & 4 & 4 & & 24974844180 & 74924815680 & 99899659860 & 124874504040 \\
\hline 17 & 5 & 4 & & 89909562600 & 269729266950 & 359638829550 & 449548392150 \\
\hline 16 & 6 & 4 & 4 & 254743928010 & 764232710670 & 1018976638680 & 1273720566690 \\
\hline 15 & 7 & 4 & 4 & 582272004600 & 1746817558200 & 2329089562800 & 2911361567400 \\
\hline 14 & 8 & 4 & 4 & 1091760220980 & 3275282619180 & 4367042840160 & 5458803061140 \\
\hline 13 & 9 & 4 & 4 & 1698293797200 & 5094884094300 & 6793177891500 & 8491471688700 \\
\hline 12 & 10 & 4 & 4 & 2207782080504 & 6623349052320 & 8831131132824 & 11038913213328 \\
\hline 11 & 11 & 4 & 4 & 2408489532540 & 7225471840860 & 9633961373400 & 12042450905940 \\
\hline 16 & 5 & 5 & 4 & 305692744500 & 917079275970 & 1222772020470 & 1528464764970 \\
\hline 15 & 6 & 5 & 4 & 815180909400 & 2445544478520 & 3260725387920 & 4075906297320 \\
\hline 14 & 7 & 5 & 4 & 1746816528600 & 5240452159800 & 6987268688400 & 8734085217000 \\
\hline 13 & 8 & 5 & & 3056929195320 & 9170791009380 & 12227720204700 & 15284649400020 \\
\hline 12 & 9 & 5 & 4 & 4415564593440 & 13246697924460 & 17662262517900 & 22077827111340 \\
\hline 11 & 10 & 5 & 4 & 52986776 & 15896037401244 & 21194715021480 & 26493392641716 \\
\hline 14 & 6 & 6 & 4 & 2037952676760 & 6113860672920 & 8151813349680 & 10189766026440 \\
\hline 13 & 7 & 6 & 4 & 4075905713880 & 12227721225720 & 16303626939600 & 20379532653480 \\
\hline 12 & 8 & 6 & 4 & 6623347190460 & 19870046376180 & 26493393566640 & 33116740757100 \\
\hline 11 & 9 & 6 & 4 & 8831129727420 & 26493395308380 & 35324525035800 & 44155654763220 \\
\hline 10 & 10 & 6 & 4 & 971424286 & 29142734424804 & 38856977287128 & 48571220149452 \\
\hline 12 & 7 & 7 & 4 & 7569539697720 & 22708624618680 & 30278164316400 & 37847704014120 \\
\hline 11 & 8 & 7 & 4 & 11354309906940 & 34062936567660 & 45417246474600 & 56771556381540 \\
\hline 10 & 9 & 7 & 4 & 3877490086460 & 41632477826940 & 55509967913400 & 69387457999860 \\
\hline 10 & 8 & 8 & 4 & 9970 & 037290 & 62448713587260 & 78060890137230 \\
\hline 9 & 9 & 8 & 4 & 73468628 & 52040597058450 & 69387459891750 & 86734322725050 \\
\hline 15 & 5 & 5 & 5 & 978217153080 & 2934653312460 & 3912870465480 & 4891087618560 \\
\hline 14 & 6 & 5 & 5 & 2445543345960 & 7336632817800 & 9782176163760 & 12227719509720 \\
\hline 13 & 7 & 5 & 5 & 4891087000800 & 14673265326720 & 19564352327520 & 24455439328320 \\
\hline 12 & 8 & 5 & 5 & 5840 & 23844 & 31792072532220 & 39740089449060 \\
\hline 11 & 9 & 5 & 5 & 597355889120 & 31792074153840 & 42389430042960 & 52986785932080 \\
\hline 10 & 10 & 5 & 5 & 657091802392 & 34971281244918 & 46628373047220 & 58285464849612 \\
\hline 13 & 6 & 6 & 5 & 706268287720 & 17118809427720 & 22825077715440 & 28531346003160 \\
\hline 12 & 7 & 6 & 5 & 597356009240 & 31792074033720 & 42389430042960 & 52986786052200 \\
\hline 11 & 8 & 6 & 5 & 896034374220 & 47688110690220 & 63584145064440 & 79480179438660 \\
\hline 10 & 9 & 6 & 5 & 9428486697620 & 58285468381140 & 77713955078760 & 97142441776380 \\
\hline 11 & 7 & 7 & 5 & 3166896427680 & 54500697931680 & 72667594359360 & 90834490787040 \\
\hline 10 & 8 & 7 & 5 & 979483308780 & 74938458935340 & 99917942244120 & 124897425552900 \\
\hline 9 & 9 & 7 & 5 & 7754981254000 & 83264954572800 & 111019935826800 & 138774917080800 \\
\hline 9 & 8 & 8 & 5 & 224354361200 & 93673073443950 & 124897427805150 & 156121782166350 \\
\hline 12 & 6 & 6 & 6 & 2363582073980 & 37090752699000 & 49454334772980 & 61817916837540 \\
\hline 11 & 7 & 6 & 6 & 194712739200 & 63584147346720 & 84778860085920 & 105973572825120 \\
\hline 10 & 8 & 6 & 6 & 9142730556940 & 87428201640780 & 116570932197720 & 145713662754660 \\
\hline 9 & 9 & 6 & 6 & 32380811767500 & 97142446701300 & 129523258468800 & 161904070223700 \\
\hline 10 & 7 & 7 & 6 & 33305977985280 & 99917945006880 & 133223922992160 & 166529900977440 \\
\hline 9 & 8 & 7 & 6 & 41632472781900 & 124897430958300 & 166529903740200 & 208162376522100 \\
\hline 8 & 8 & 8 & 6 & 6836532142400 & 140509609039800 & 187346141182200 & 234182673324600 \\
\hline 9 & 7 & 7 & 7 & 7579968893600 & 142739921095200 & 190319889988800 & 237899858882400 \\
\hline 8 & 8 & 7 & 7 & 53527465605900 & 160582410631500 & 214109876237400 & 267637341843300 \\
\hline
\end{tabular}


